MEASUREMENT OF THE COMPLEX CONDUCTIVITY OF AN IONIZED GAS AT MIOROWAVE FREQUENCIES

Thesis by

Fred P. Adlex

In Partial Fulfillment of the Requirements For the Degree of Doctor of Philosophy

California Institute of Technology Pasadena, California 
ACKNOWLEDGMENT

I am indebted to Dr. M.D. Fiske of the Geneal Electrio Research Laboratory who suggested a simiIar study in 1946, and to Dr. J.D. Cobine, of the same laboratory, with whose help this project was initiated. The bulk of the woxk was done at the California Institute of Technology, under the helpw ful and sympathetic guidance of Professors W. H. Pickering and S.S. Mackeown. To all the members of the staff I shall always be indebted for the excellent instruction and inspiration they have provided which have made my stay at the Institute both profitable and pleasant.

Financial assistance was generously provided by the General Electric Educational Fund in the form of two Charles A. Cofin Fellowship grants. 
ABSTRACT

The positive colum of a glow discharge is placed along the axis of a cylindrical cavity excited in the TMO10 mode. The transmission of $3 \mathrm{~cm}$ waves through the cavity and the shift in resonant frequency are observed as a function of disw charge current. It is shown that from these measurements values of the complex conductivity of the electron gas can be calculated. Curves of the measured conductivity components as functions of pressure and current are given. From theoretical expressions for the conductivity values of electron density are calculated. Langmuir probe studies are carried out and adequate agreement is found. Finally, mean free path and mean free time between collisions are calculated from the conducm tivity values and hence the variation of collision cross-secm tion with temperature is found. 
TABLE OF CONTENTS

TIPLE

PAGE

I. INTRODUCTION . . . . . . . . . . . I

II. EXPERIMENTAL TECHNIQUE AND APPARATUS ..... 2

III. THEORETICAL RELATIONS ......... 5

A. Nomenclature ........... 5

B. Calculation of the Real Component of Conductivity ............

C. Calculation of the Imaginary Component of Conductivity ........... 10

D. Theoretical Expressions for Conductivity • 14

E. Miscellaneous Formulas ........ 22

IV. EXPERIMENTAL RESULTS AND DISCUSSION • . . . 26

V. FIGURES .............. 30

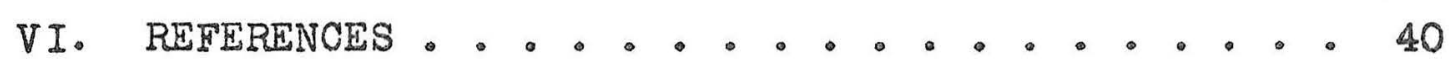

VII. APPENDIX (Publications)............ 43 


\section{INTRODUCTION}

A knowledge of the conductivity of an ionized gas is of interest in several phases of microwave work, such as the design of TR tubes (I), as weIl as in many problems connected with the study of the ionosphere (2); it has also acquired considerable importance in guided missile work, since the conductiv ity of the rocket motor flame may cause malfunctioning of the missile-ground radio link $(3,4,5)$. Some work has been done on the behavior of gaseous discharges at microwave frequencies (615), but published measurements of $r . f$ conductivity $(16,17,18)$ are restricted to lower frequency ranges.

A determination of conductivity at a wave length near $3 \mathrm{~cm}$ is described bere. A d.c. mercury vapor glow discharge was used to supply the conducting gas which had the advantage that eleco tron temperature and density could be determined by probe studies, using Langmuir's theory (19). In conjunction with a theoretical equation for conductivity (20) these measurements allow one to make a check of the values for the conductivity obtained experimentally.

A study is in progress at present at the C.I.T. Jet Propulsion Laboratory to determine the conductivity of various jet flames, using the method described in this thesia. 
II. EXPERIMENTAL TECHNIQUE AND APPARATUS

At this writing the use of a resonant cavity as a means of applying a microwave field to a discharge is no longer a novel idea. Various resonant structures have been used lately to measure the dielectric constant of gases (21-28) and to study breakdown and diffusion phenomena $(9,11-15)$. The method used in the references cited is based on finding the cavity input admittance by the usurl standing-wave ratio and phase measurements: to determine complex conductivity in this way leads to rather involved problems (ef. reference $29, \mathrm{pp} .22$ and 32). The scheme described below is a transmission, rather than a reflection method, and allows a relatively simple and straightforward determination of conductivity to be made.

A schematic diagram of the experimental arrangement is shown in Fig. 1. A cylindrical cavity was used (Fig. 2), designed to resonate near a wave length of $3 \mathrm{~cm}$ in the TMOlO mode. Coupling to the wave guides was through circular irises in the side walls. Small openings at the centers of the top and bottom walls allowed the discharge tube to extend axially through the cavity; since/the mode used the cavity wall currents fall to zero at the centers of the end plates, these apertures have no appreciable disturbing effect. This was not immediately recognized when this research was started, and early experiments were made with a cavity using radial chokexs designed to prem sent a short circuit to $3 \mathrm{~cm}$. waves and an open circuit to d.c. (cf. reference $30, p .359$ ). In this way the top plate could 
be insulated from the rest of the cavity and the discharge then took place between the top and bottom cavity walls. D.c. breakm down between the closely spaced radial chokefs gave considerable trouble; moreover, the $Q$ of this cavity was only about 700 , while $a$ Q $Q$ of about 3000 was obtained with the simpler cavity shown in Fig. 2. Use of radial chokexs was thence abandoned. A quartz tube, shown in Fig. 2 , was used to contain the glow discharge, after it had been found that even very thinwalled pyrex tubes loaded down the cavity too much due to the relatively bigh loss tangent of glass. Insertion of the quartz tube reduced the cavity $Q$ only by a few percent although its high dielectric constant shifted the resonant wave length from $3 \mathrm{~cm}$. to $3.55 \mathrm{~cm}$. The bottom part of the tube was formed into a bulb containing a small pool of distilled mercury. In the experiment this bulb extended into an oilbath the temperature of which was thermostatically controlled to $\pm \frac{1}{2}{ }^{\circ} \mathrm{C}$ and could be varied from room temperature to slightly above $200^{\circ} \mathrm{C}$. The rew maining portion of the tube, containing the actual discharge, was placed in an oven the temperature of which was maintained about $25^{\circ} \mathrm{C}$ above that of the oilbath in order to prevent unwanted condensation from occurring. Thus all condensation ocm curred at the surface of the mercury pool whose temperature then determined the pressure of the mercury vapor once equilibrium had been established. Since the tube had been carefully evacuated, the mercury vaporpressure was also the total pressure in the tube which could thus be determined by measuring the temperature of the oil bath. 
The high frequency excitation was obtained from a klystron tube frequency-modulated at a 1000-c.p.s. rate by superposing a small sawtooth voltage on the repeller potential; the same modulation was also applied to the horizontal sweep of an oscilloscope. The output from the cavity was picked up by a crystal, amplified, and applied to the vertically deflecting plates of the oscilloscope. The resulting trace was thus a Q-curve, whose height was proportional to the transmission in power through the cavity since a square law crystal was used. A calibrated weve meter placed between cavity and crystal introduced small "pips" on the trace by means of which the resonant frequency and the $Q$ of the cavity could be measured. As shown below, measurement of these three quantitieg- effective $Q$, change in transmission,

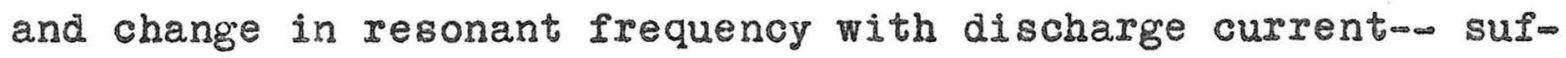
fices to determine both real and imaginary cormponents of the conductivity.

For the purpose of carrying out probe studies a second disw charge tube was built identical to the one used in the cavity, except for a fine probe wire extending radially into the middle portion. 
III. THEORETICAI RELATIONS

A. Nomenclature

t.......transmission in power through the cavity, i.8., ratio of output power from the cavity to available power. tr......elative transmission, i.\&., ratio of transmission $t$ with discharge current flowing to zero-ourrent transw mission.

a........ radius of cavity $(1.15 \mathrm{~cm})$.

h.......height of cavity $(1.5 \mathrm{~cm})$.

b........ radius of conducting column $(0.175 \mathrm{~cm})$.

$\sigma_{r}+j \sigma_{i} \ldots$ complex conductivity of ionized mercury vapor, mhos/meter.

Q $0 \ldots . .$. the $Q$ of the unloaded cavity. $Q_{L} \ldots . .$. of $^{2}$ cavity with loading due to coupling to the externo al circuit through the irises. $Q_{1} \ldots . .$. an iris loading $Q$ defined by $1 / Q_{L}=1 / Q_{0}+1 / Q_{i}$. Q $\ldots . . . Q$ of cavity loaded by the discharge (but not by Irises). wo......nominal resonant frequency of the cavity, radians/second. w.......resonant frequency with discharge current flowing.

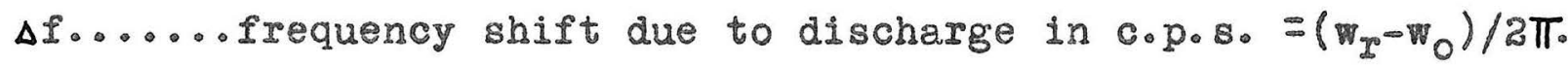
p.......vapor pressure, mm Hg。

T.......electron temperature, ${ }_{\mathrm{K}}$. n.....electron density, electrons/ $\mathrm{cm}^{3}$. $x_{1} \ldots . . . a$ dimensionless parameter $=\left(m w^{2} \lambda^{2} / 2 \mathrm{kT}\right), \lambda$ being the electronic mean free path; for mercury at 8460 Mo: $\mathrm{x}_{1}=1.2 \times 10^{6} \mathrm{p}^{-2} \mathrm{~T}^{-1}$. 


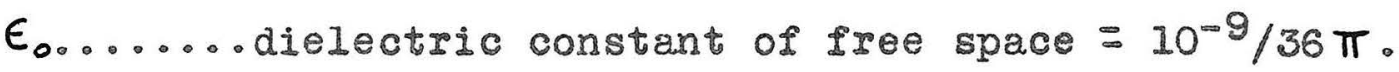

$\epsilon_{1} \ldots \ldots \epsilon_{0}\left(1 \rightarrow \sigma_{i} / w \epsilon_{0}\right) ;$ ci. Eq. (8).

$\mathrm{k}_{1} \ldots \ldots \mathrm{w}_{\mathrm{r}}\left(\mu \epsilon_{1}\right)^{\frac{1}{2}}$

$k_{2} \ldots \ldots w_{r}\left(\mu \epsilon_{0}\right)^{\frac{1}{2}}$.

k........2. $405 / 2$.

B........equivalent shunt susceptance of the cavity.

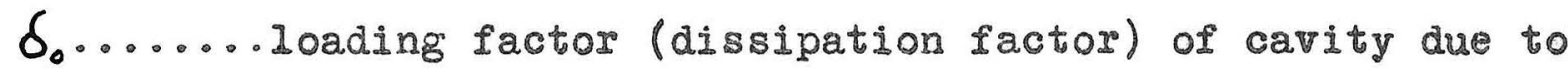
the Iinite conductivity of the walls; i.e., ratio of equivalent conductance to equivalent susceptance.

(Note: $\left.\delta_{0}=1 / Q_{0}\right)$.

$\delta_{1} \ldots \ldots . . .$. input loading factor of cavity; i.e. ratio of conductance coupled to cavity from input end to the shunt susceptance of the cavity.

$\delta_{2} \ldots \ldots . . .0 u t p u t$ loading factor of cavity.

$\delta_{L} \ldots . . . .$. loading factor of cavity with irises $=1 / Q_{L}$.

J......... current density (amperea per sq. meter).

$\lambda \ldots \ldots$......electronic mean free path.

M........mass of neutral mercury atom.

N........mercury atoms pex $\mathrm{cm}^{3}$.

K......electron mobility.

U........drift speed of electrons in a d. c. field $\left(u=K{ }^{\prime}\right)$.

T.......mean free time of electrons between collisions.

q..........ectronic collision cross-section.

マ.......electron velocity

po.......pressure at which $\sigma_{r}$ reaches a maximum (mm $\mathrm{Hg} \cdot$ ). A.........probe area exposed to the discharge.

i.........electron current collected by the probe. 
id.......discharge current.

P........power dissipated in the cavity.

(Note: Except where expressly noted otherwise, all formulas are in rationalized mok。s. units.)

\section{B. Calculation of the Real Component of Conductivity}

An expression for $t$, the transmission in power through the cavity, will be derived fixst. For this purpose the cavity is assumed to be at or near the dominant resonant mode (TMO10), so that it can be represented by a shunt resonant circuit to which are coupled resistive input and output circuits as shown in the accompanying sketch.

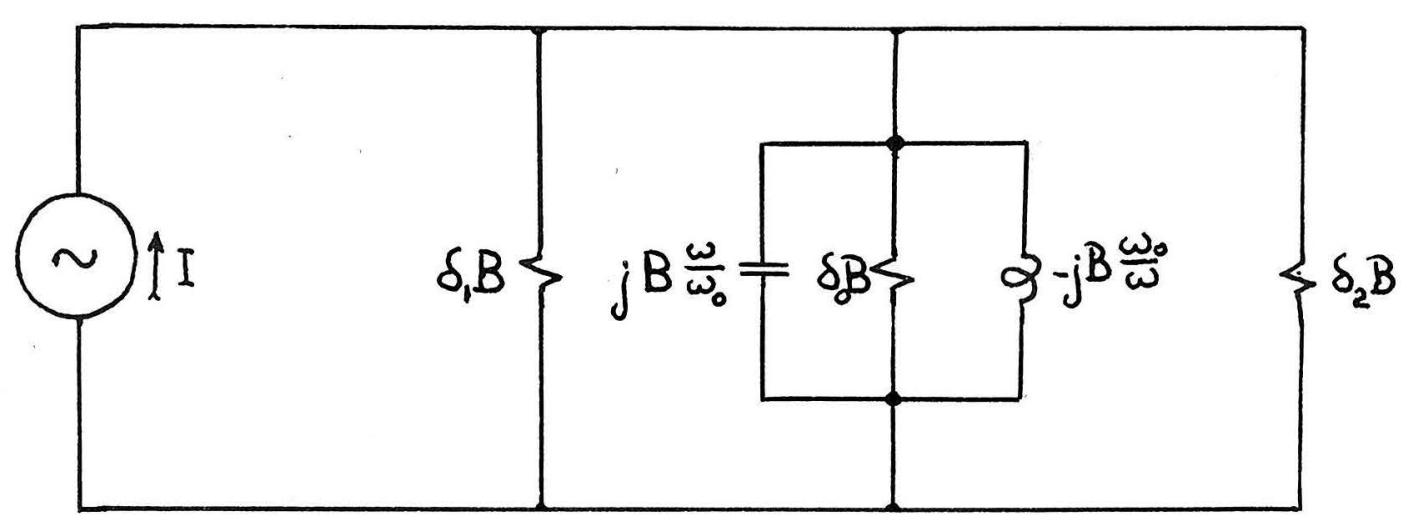

EQUIVALENT SHUNT RESONANT CIRCUIT

When the cavity is excited by energy supplied from the input circuit there exists in the cavity a certain amount of reactive power, a fraction $\delta_{0}$ of which is dissipated as losses in the cavity itself; note that $\delta_{0}=1 / Q_{0}$. The symbol $Q_{0}$ is defined bere as the intrinsic $Q$, i.e., the $Q$ without any external load- 
ing, to differentiate it from the more general $Q_{L}$ which is the measured $Q$ when the cavity is loaded down by external coupling. Coupling to the external circuit then increases the loaded $\delta$ to

$$
\delta_{L}=\delta_{0}+\delta_{1}+\delta_{2}
$$

Physically the assumption underlying this expression is that the distribution of electro-magnetic fields within the cavity is not seriously altered by the input and output coupling devices, an assumption which is certainly valid as long as the $\delta$ 's are very small compared to unity. This requirement was met in the actual experiment in which the $\delta^{8} \mathrm{~s}$ were of the order of $10^{-4}$.

The available power in the above circuit is that delivered in a matched condition, viz.,

$$
s_{a}=\left(\frac{I}{2 \delta_{1} B}\right)^{2} \cdot \delta_{1} B=\frac{I^{2}}{4 \delta_{1} B}
$$

while the power actually going into the Ioad $\left(\delta_{2} B\right)$ is

$$
P_{L}=\left(\frac{I}{\delta_{B} B+\delta_{1} B+\delta_{2} B}\right)^{2} \cdot \delta_{2} B=\frac{I^{2} \delta_{2}}{\delta_{L}^{2} B}
$$

The transmission then comes out to be

$$
t=\frac{P_{L}}{P_{a}}=\frac{4 \delta_{1} \delta_{2}}{\delta_{L}^{2}}
$$

Next symmetrical conditions are assumed to exist making $\delta_{1}$ equal to $\delta_{2}$ This was insured by placing smooth padding attenuators on either side of the cavity (cf. Fig. I) so that the cavity looks into $Z_{0}$, the characteristic line impedance both 
ways. If now the symbols $Q_{\mathcal{L}}$ and $Q_{1}$ are introduced, where $Q_{L} \approx I / \delta_{L}$ and $1 / Q_{L}=1 / Q_{0} \neq 1 / Q_{i}$, the transmission can be written as

$$
t=\left(\frac{Q_{i}}{Q_{i}}\right)^{2}=\left(1+Q_{i} / Q_{0}\right)^{-2}
$$

Note that for any additional internal loading of the cavity, $Q_{0}$ in (5) must be replaced by the corresponding (Iower) Q. Thus with discharge current flowing Eq. (5) becomes

$$
t=\left(1+Q_{j} / Q^{1}\right)^{-2}
$$

For the cavity loaded by wall losses only, $Q_{0}$ may be expressed in terms of an equivalent shunt resistance $R$ along the cavity axis as (3I)

$$
Q_{0}=0.00531 \frac{a}{h} R \text {. }
$$

The very small additional loading due to the presence of the quartz tube can be assumed to be lumped into $R$, giving a slightly lower effective $Q_{0}$. The conducting column is now assumed to introduce an additional shunt resistance $R^{8}$ placed in parallel with R:

$$
R^{1}=\frac{b}{\pi b^{2}} \cdot \frac{1}{\sigma_{r}}
$$

Thus the resultant $Q$ becomes now

$$
Q^{B}=0.0053 I_{L^{2}}^{a} \cdot \frac{R R^{\beta}}{R+\bar{R}^{B}}
$$

or uging (7) to substitute for $R$ :

$$
Q^{\prime}=\frac{Q_{0}}{I+188 Q_{0} \mathrm{~h} / \mathrm{R}^{0} \mathrm{a}}
$$

Substituting this expression into Eq. (6), and using (8) to write 
$R^{9}$ in terms of $\sigma_{r}$, the resultant expression may be manipulated into the following factored form:

$$
t=\left(1+Q_{1} / Q_{0}\right)^{-2}\left(1+592 \frac{b^{2}}{a} Q_{L} \sigma_{r}\right)^{-2}
$$

The first bracket is recognized as the transmission with no dism charge current flowing; using this as a base the second bracket may be defined as a relative transmission, $t_{x}$, in terms of which $\sigma_{r}$ can be expressed explicitiy as:

$$
\sigma_{r}=0.00169 \frac{\mathrm{a}}{\mathrm{b}^{2}} \frac{1}{\mathrm{QL}}\left(t_{r}{ }^{-\frac{1}{2}}-1\right) \text { mhos/meter. }
$$

$t_{I}$ is readily determined, being simply the ratio of the height of the Q-curve trace with discharge current flowing to that with the glow turned off; for convenience and accuracy a meter actually used which was calibrated to read $t_{1}$ directly. Since all quantities on the right-hand side of (12) can be measured, $\sigma_{r}$ may be found.

\section{c. Calculation of the Imaginary Component of Conductivity.}

From Maxwel1's Field equation

$$
\nabla \times \vec{H}=\frac{\partial \vec{D}}{\partial t}+\left(\sigma_{r} \otimes j \sigma_{i}\right) \vec{E}=\sigma_{r} \vec{E}+j w \epsilon_{0}\left(I+\frac{\sigma_{i}}{W \epsilon_{0}}\right) \vec{E},
$$

the out-of-phase conductivity component $\sigma_{i}$ is seen to reault in an effective dielectric constant

$$
\epsilon_{1}=\epsilon_{0}\left(1 \otimes \frac{\sigma_{i}}{W \epsilon_{0}}\right) \text {. }
$$

Since $\sigma_{i}$ can be shown to be negative, being due to the inertial lagging of the electrons behind the applied field, the dielectric constant is reduced, decreasing the equivalent capacity of the 
cavity; thus the effect of the glow discharge is to raise the resonant frequency, as shown by Eq. (19) below.

To treat the cavity as a boundary value problem, it is divided into two regions, where for $0<p<b, \epsilon_{1}=\epsilon_{0}\left(I+\sigma_{i} / w \epsilon_{0}\right)$, while for $b<\rho<a$ the dielectric constant is still $\epsilon_{0}$. No acm count is taken of the narrow region filled by the quartz tube which introduces a small constant frequency shift. For the in ner region the expressions for the fields will be of the same form as for the usual TMO10 mode (cf. reference 30, p. 397):

$$
E_{Z 1} \approx J_{0}\left(k_{I} \rho\right), \quad H_{\varphi I}=\left(w \epsilon_{1} / k_{1}\right) J_{1}\left(k_{1} \rho\right) \text { 。 }
$$

In the outer region Bessel functions of the second $\mathrm{kind}$ need also be included, so that for $b<\rho<a$ :

$$
\begin{aligned}
& E_{Z 2}=A_{0} J_{0}\left(k_{2} p\right)+B Y_{0}\left(k_{2} p\right) \\
& H_{\varphi 2}=\left(w \epsilon_{0} / k_{2}\right)\left[A J_{1}\left(k_{2} p\right)+B Y_{1}\left(k_{2} p\right)\right]
\end{aligned}
$$

Three boundary conditions apply: at $\rho=a, \mathbb{E}_{z Z}=0$, since the tangential electric field must vanish at the surface of a cono ductor; at $\rho=b, E_{z Z}=E_{z 1}$ and $H_{\varphi 2}=H_{\varphi 1}$ from the continuity of tangential fields. Using two of these relations to eliminate the constants $A$ and $B$, the third one may be used to obtain the following equation:

$$
\frac{J_{1}\left(k_{2} b\right) Y_{0}\left(k_{2} a\right)-J_{0}\left(k_{2} a\right) Y_{1}\left(k_{2} b\right)}{J_{0}\left(k_{2} b\right) Y_{0}\left(k_{2} a\right)-J_{0}\left(k_{2} a\right)-Y_{0}\left(k_{2} b\right)}=\left(\frac{\epsilon_{1}}{\epsilon_{0}}\right)^{\frac{1}{2}} \frac{J_{1}\left(k_{1} b\right)}{J_{0}\left(k_{1} b\right)}
$$

The desired new resonant frequency is now contained implicitly in $\mathrm{k}_{2}$ and $\mathrm{k}_{1}$, and is solved for by plotting each side of (16) seperately over a small range near $w=w_{0}, \epsilon_{1}=\epsilon_{0}, \mathrm{k}_{1}=\mathrm{k}_{2}$ 。 
From these curves the following linear approximations may be obtained:

$$
\begin{aligned}
& \frac{J_{1}\left(k_{1} b\right)}{J_{0}\left(k_{1} b\right)} \approx 0.1860+0.1 .940\left[\left(\frac{\epsilon_{1}}{\epsilon_{0}}\right)^{\frac{1}{2}} \frac{W_{x}}{W_{0}}-1\right] \\
& \Phi\left(k_{2} b, k_{2 a}\right) \cong 0.1860-4.388\left[\frac{W_{x}}{W_{0}}-1\right]
\end{aligned}
$$

where $\Phi\left(\mathrm{k}_{2} \mathrm{~b}, \mathrm{k}_{2} \mathrm{a}\right)$ has been written for the left hand side of Eq. (16). Since the change in $\epsilon$ due to $\sigma_{i}$ is of the order of only 0.5 percent, one may wite

$$
\left(\frac{\epsilon_{1}}{\epsilon_{0}}\right)^{1 / 2}=\left(1+\frac{\sigma_{i}}{w \epsilon_{0}}\right)^{1 / 2} \cong 1+\frac{\sigma_{i}}{2 \omega \epsilon_{0}}
$$

From a substitution of Eqs. (17) and (18) into Eq. (16) the folIowing first-order approximation is finally obtained for the particular case at hand:

$$
\sigma_{i}=-1.33 \times 10^{-9} \times \Delta f \quad \text { mhos/meter }
$$

The relation between $\sigma_{i}$ and frequency shift may be derived more simply by an alternate method which makes use of the variation principle for electromagnetic problems, vizon that at resonance

$$
\text { time } \int_{\text {volume }} \int\left(\frac{1}{2} \mu \mathrm{H}^{2}-\frac{1}{2} \in \mathbb{E}^{2}\right) d v d t
$$

have a stationary value of zero. This is analagous to Hamilton's principle in mechanics, the volume integrals of $\frac{1}{2} \mu \mathrm{H}^{2}$ and $\frac{1}{2} \in \mathbb{E}^{2}$ representing kinetic and potential energies respectively. Since all time variations are sinusoidal, and $\mathrm{H}$ may be replaced from Faxaday's Law, $\nabla \times E=-j w \mu H$, this relation can be rewritten as 


$$
w_{r}^{2}=\frac{\int_{v o l} \frac{1}{\mu}(\nabla \times E)^{2} d v}{\int_{v o l} \epsilon E^{2} d v}
$$

If the perturbation of the electric field due to the discharge is not appreciable, only the denominator of this equation will cause any change in the resonant frequency since $\epsilon$ is reduced in accordance with Eq. (13). Thus the effect on the resonant frequency of any small changes in the dielectric constant can be expressed by

$$
\frac{W_{x}}{W_{0}}=\frac{\int_{v 0 l} \epsilon_{0} E^{2} d v}{\int_{v o l} \epsilon E^{2} d v}
$$

Where in this case $\epsilon$ is given by Eq. (13) over the region $0<p<b$. It is seen that $w_{x}$ is most sensitive to changes in $\epsilon$ which occur in regions of high electric field strength which is exactly the case here since the discharge tube is placed along the cavity axis where E reaches a maximum. FinalIy the square root may be expanded as in Eq. (18), so that (22) becomes

$$
\frac{w_{p}-w_{e}}{w_{0}}=-\frac{\sigma_{L}}{2 w_{r} \epsilon_{0}} \frac{\int_{0}^{b} E^{2} p d p}{\int_{0}^{a} E^{2} p d p}
$$

Taking $E$ to be proportional to $J_{0}(k p)$ as in the undisturbed case, integration yields the following result:

$$
\Delta f=-\frac{\sigma_{i}}{4 \pi \epsilon_{0}}\left(\frac{b}{g}\right)^{2} \frac{J_{0}^{2}(k b)+J_{1}^{2}(k b)}{J_{1}^{2}(k a)}
$$

Making the appropriate numerical substitutions Eq. (24) is 
found to agree within one percent with Eq. (19).

\section{Theoretical Expressions for Conductivity}

If a high frequency electric field is applied to an ionized gas, the resulting oscillatory motion of the free electrons will give rise to an alternating current. In general this current will not be in phase with the electric field; if it is written a

$$
J=\sigma \mathbb{E}
$$

Then this behavior may be described by a complex conductirity $\sigma=\sigma_{r}+j \sigma_{i}$. Two limiting cases for $\sigma$ are well know: for high frequencies and low pressures there is practically no enexgy loss due to electron collisions and hence the current is in quadrature with the field $\left(\sigma_{r}=0\right)$. The equation of motion of an electron under these circumstances is given by

$$
m \ddot{z}=e E=e E_{0} \exp (J w t)
$$

for an electric field in the $z$ direction. The current density is then simply

$$
J=n e^{i}=n e\left(\frac{e E}{J w 2 z}\right)=-j \frac{n e^{2}}{w n}
$$

whence the conductivity is, by Eq. (25):

$$
\sigma=-j \frac{n e^{2}}{w m} \text { or } \quad \sigma_{i}=-\frac{n e^{2}}{w m}
$$

The other limiting case occurs at low frequencies and high pregm wres in which case the average velocity of the electrons, $\dot{z}_{\text {, }}$ 
is simply related to the electric field by the electron mobility, $\mathrm{K}$, so that

$$
J=\text { néz }=\text { neKE }
$$

or

$$
\sigma=\sigma_{r}=n e X=\frac{3 \sqrt{2 \pi}}{16} \frac{e^{2} \lambda n_{1}}{(\operatorname{mkT})^{\frac{1}{2}}}
$$

where Langevin's mobility formula (32) has been made use of in the last expression.

In order to treat the more general intermediate case it is first necessary to find the velocity distribution function for the free electrons. Let $f\left(v_{x}, v_{y}, v_{z}\right) d v_{x} d v_{y} d v_{z}$ be the number of electrons having velocity components about $\nabla_{X}, \nabla_{y}, \nabla_{Z}$, falling within the element $d v_{x} d v_{y} d v_{z}$ (or $v^{2} \sin \theta d \varphi d \theta d v$ ) in velocity space; $f$ is not a function of the coordinates (in physical space) since diffusion and similar phenomena are disregarded here, the electron concentration $n$ being assumed uniform over the volume considered. The function $f$ is detexmined from Boltzmann's transfer equation (33):

$$
\frac{e E}{m} \frac{\partial f}{\partial v_{z}}+\frac{\partial f}{\partial t}=\left[\frac{\partial f}{\partial t}\right]_{e}
$$

Here $\frac{\partial f}{\partial t}$ represents a smooth time rate of change caused by the variations in the electric field $E$ which is applied in the $z$ direction; $\left[\frac{\partial f}{\partial t}\right]_{e}$ represents the rate of change occasioned by elastic collisions, in-elastic collisions being neglected since the electric field is assumed to be relatively weak. The general procedure now, following Lorentz $(34,35)$, is to expand $f$ in a series of Legendre functions in $\cos \theta=\mathrm{v}_{z} / \mathrm{v}$ : 


$$
\begin{aligned}
& \mathcal{I}\left(\boldsymbol{v}, v_{z}\right)=f_{0}(v)+p_{1}(\cos \theta) f_{1}(v)+p_{2}(\cos \theta) \mathfrak{I}_{2}(v) \ldots \ldots \\
& =f_{0}(v) \propto\left(v_{Z} / v\right) f_{I}(v)+\ldots
\end{aligned}
$$

The first term determines the random distribution in velocity, while the second term represents the electron "drift" in response to the applied field. Higher terms do not correspond to any simple physical property of the distribution and are neglected. In order to carry through the solution of $\mathrm{Eq}$. (31) using Eq. (32) it is found necessary to make one of two simplifying assumptions: the electronic mean free path, $\lambda$, is independent of the electronic velocities; or the mean free time, $\tau$, between collisions is constant. The meaning of these two hypotheses is perhaps best seen by considering the general relation

$$
\mathbb{N} q \lambda=1
$$

where $N$ is the number density of obstacles (i.e., mercury at oms in this case), $q$ the electronic collision cross-section which is ir general a function of the electron velocity $v$. Constant $\lambda$ thus implies constant $q$. on the other hand, since $\tau=\lambda / v$, constant $\tau$ would mean that $q$ is inversely proportional to $v$. The actual behavior of $q$ has been the subject of considerable research (36). It has been found experimentally (37) that for the monatonic gases that are the vapors of the metals, q first decreases rapidly with velocity and then remains quite constant at high electron temperatures. As will be shown in section IV, the results of this investigation show that $\lambda$ and $q$ do not change over the range of electron temperatures encountered. An expression for complex conductivity has been derived by H. Nargenau $(20,38,39)$ which is exact for the case in which 
$\lambda$ does not vary with $v$ ( $i, e, p \lambda$ equals a constant, independent of the electron temperature). He writes Eq. (32) in the form

$$
f=f_{0}(v)+(v z)\left[F_{1}(v) \cos w t \leftrightarrow G_{1}(v) \sin w t\right]
$$

since the applied field, $E_{0} \cos w t$, will induce the corresponding frequency in the $f_{1}(v)$ of $\mathrm{Eq}$. (32) which, in general, will have one part in phase with the field and another part out of phase. Margenau then shows that $f_{0}$ has a Maxwellian distribution,

$$
f_{0}=n(m / 2 \pi k T)^{3 / 2} \exp \left(-\frac{m v^{2}}{k K T}\right)
$$

provided the a.c. field is not too strong; specifically if

$$
\mathrm{E}_{0} \leq \frac{\mathrm{mw}}{\mathrm{e}} \sqrt{\frac{6 \mathrm{kT}}{\mathrm{H}}}
$$

He further finds that

$$
\begin{aligned}
& G_{1}=\frac{w \lambda}{v} F_{1} \\
& f_{1}=-\frac{\lambda}{v+j \omega \lambda} \frac{\partial f_{0}}{\partial v} \frac{e E}{m}
\end{aligned}
$$

Knowing $f_{1}$, the complex conductivity is obtained in a manner analagous to that employed below for the case of constant $\tau$. Margenau's final result for the complex conductivity is

$$
\sigma=\frac{4}{3} \frac{e^{2} \lambda_{n}}{\left(2 \pi m k^{T}\right)^{\frac{1}{2}}}\left[x_{2}\left(x_{1}\right)-j \sqrt{x_{1}} K_{3 / 2}\left(x_{1}\right)\right]
$$

where

$$
K_{n}\left(x_{1}\right)=\int_{0}^{\infty} \frac{x^{n} e^{-x}}{x+x_{1}} d x
$$

$\mathrm{K}_{2}$ and $\mathrm{K}_{3 / 2}$ have been plotted by Margenau. For $\mathrm{x}_{1} \rightarrow \infty$ and $\mathrm{x}_{1} \rightarrow 0$ the above expression approaches the two limiting cases given in Eqs. (28) and (30), although in the latter case the numerical 
factors differ by about ten percent. For mercury vapor at 8460 Mc, the effective resonant frequency of the cavity with the tube inserted, the above expression becomes*:

$$
\sigma=4.38 \times 10^{-10}\left(\mathrm{n} / \mathrm{pT}^{\frac{1}{2}}\right) \mathrm{K}_{2}\left(\mathrm{x}_{1}\right)-\mathrm{j} 4.81 \times 10^{-7}\left(\mathrm{n} / \mathrm{p}^{2} \mathrm{~T}\right) \mathrm{K}_{\frac{3}{2}}\left(\mathrm{x}_{1}\right)
$$

A plot of $\sigma$ VB. pressure at constant frequency shows that $\sigma_{i}$ decreases monotonically, starting with the value $e^{2} n / m w$ at very low pressures and going to zero as we ${ }^{2} \lambda / 3 \mathrm{kT}$ at bigh pressures; the real component of the conductivity falls to zero at both very low and very high pressures; a maximum is predicted (17) for:

$$
w \lambda=6.8 \times 10^{13}(\mathrm{kT})^{\frac{1}{2}} \quad\left(\mathrm{cog} . \mathrm{s}_{0}\right)
$$

A more convenient form of this equation is the following:

$$
p_{0}^{2} T=570,000
$$

Here $p_{0}$ is the pressure in $\mathrm{mm}$ Hg at which $\sigma_{r}$ maximizes.

It may now be of interest to carry through a treatment analagous to Margenau's, but considering $\tau$ to be constant. Note that

$$
\tau=\frac{1}{\xi}=\frac{\lambda}{v}
$$

where $\zeta$ is the average collision frequency and $v$ the electron velocity; the fact that $\lambda / v$ is independent of $v$ will be seen to simplify this derivation. Now a.c. current density $J=n e \bar{v}_{z}$ where $\bar{\nabla}_{2}$ is the instantaneous average drift velocity of the 
electrons given by the average over all velocity space:

$$
\bar{v}_{z}=\frac{1}{n} \int_{0}^{\infty} \int_{0}^{\pi} \int_{0}^{2 \pi} v_{z^{f}} v^{2} d v \sin \theta d \theta d \varphi
$$

The distribution function $f$ is given by Eq. (32), but only the second term, $\left(v_{z} / v\right) f_{I}(v)$, is used here since $f_{0}(v)$ does not contribute to the a.c. current density. Writing $v_{z}=v \cos \theta$ in the integrand, the integrations over polar angle $\theta$ and arimuth $\varphi$ in velocity space may be performed, leaving

$$
\bar{v}_{z}=\frac{4 \pi}{3 n} \int_{0}^{\infty} f_{I}(v) v^{3} d v
$$

If now $f_{I}$ is replaced from Eq. (38), the current density becomes

$$
J=n e v_{z}=-\frac{4 \pi e^{2} E}{3 m} \int_{0}^{\infty} \frac{(\lambda / v)}{1+j w(\lambda / v)} \cdot \frac{\partial \varepsilon_{0}}{\partial v} v^{3} d v
$$

Since $\tau=\lambda / v$ is not a function of $v$ in this treatment, the integration is readily carried through:

$$
\int_{0}^{\infty} \frac{\partial f_{0}}{\partial v} v^{3} d v=\left.v^{2} f_{0}\right|_{0} ^{\infty}-3 \int_{0}^{\infty} f_{0} v^{2} d v
$$

The first term is zero at both limits for all physical distribution functions. The second term has the value $3 n / 4 \pi$ quite independently of the actual form of $f_{0}$; this follows from the fact that the distribution function must be normalized to the electron concentration, i.e.,

$$
n=4 \pi \int_{0}^{\infty} f_{0} v^{2} d v
$$


Upon rationalizing Eq. (45), the resulting expression for the conductivity becomes simply:

$$
\sigma=J / E=\frac{e^{2} n}{m} \frac{(I-j w \tau) \tau}{I+w^{2} \tau^{2}}
$$

It is of interest to note here that this or a very similar result could have been obtained by a much simpler, though less rigorous method. The equation of motion of an electron in an alternating field may be written as

$$
m \ddot{z}+g \dot{z}=e E_{O} \exp (j w t)
$$

where $g \dot{z}$ is a friction term representing the effect of repeated collisions. This simple equation allows the conductivity to be found immediately:

$$
\sigma=n e \dot{z} / E=\frac{n e^{2}}{j w m+g}=\frac{1-j \frac{m}{I+\left(\frac{m}{b}\right)}}{g} \frac{e^{2} n}{g}
$$

Hulbert (40) and others have used a suggestion of Lorentz to the effect that $g=2 m / \tau$. The justification for this expresm sion is seen readily if one considers the motion of an electron in a steady field where inertia effects do not enter. Then the average drift speed, $u$, will be such that $g u=e E ;$ but the drift speed is also related to $E$ by the electron mobility, $K$ :

$$
U=K E=c \frac{e \lambda}{m \bar{V}} E=c \frac{e E}{m} \tau
$$

Here $\bar{V}$ denotes the average thermal velocity and $c$ is a numerical factor varying between 0.5 and 1.0 depending on the assumptions made in the statistical analysis of the motions of the electrons and molecules (see reference $36, \mathrm{pp}, 600$ et seq.). If all 
electrons are assumed to have identical free paths and thermal velocities, then c obviously equals one half, since $(\mathrm{eE} / \mathrm{m}) \tau$ is the terminal velocity just prior to a collision which is here assumed to destroy all momentum in the direction of the field; hence $g=m / c \tau=2 m / \tau$. A somewhat more realistic derivation which takes into account the distribution of free paths (reference 36, p. 47) yields $c=1$ and thus $g=m / \tau$. If this is now substituted into Eq. (50), Eq. (48) is obtained. The exact agreement is, of course, rather fortuitous, for more precise derivations of $K$ using the elastic collision theory of Langevin $(41,42)$ give a value of about 0.75 for $c$.

The behavior of $\sigma$ with changes in frequency or pressure predicted by Eq. (48) is quite similar to that found from Eq. (39). The real component of conductivity reaches a maximum now when

$$
w \tau=1
$$

or when

$$
{ }_{w} \lambda=\bar{v}=(8 \mathrm{kT} / \pi \mathrm{m})^{\frac{1}{2}}=5.29 \times 10^{13}(\mathrm{kT})^{\frac{1}{2}},(\mathrm{c} \cdot \mathrm{g} \cdot \mathrm{s})(53)
$$

a result not very different from Eq. (4I). Both equations are seen to place the maximum of conductivity nearly at that value of the pressure for which the mean free time between impacts is equal to a half-period of the a.c. field; such a result is expected since $\sigma_{r}$ reaches a maximum when electron collisions take the most energy out of the field, i.e., when the electrons are allowed to acquire a maximum of kinetic energy between collisions. 


\section{E. Miscellaneous Formulas}

1. Probe Studies*

In order to make possible a comparison of measurement and theory it was necessary to determine two parameters of the gas discharge which enter into the equation for conductivity, nameIy the electron temperature and the electron density. Follow ing Langmuir, the procedure used was essentially one of varying the potential of a small probe wire extending into the plasma and studying the probe current as a function of the probe voltage.

Now Boltzmann's relation shows (43) that over a certain region of probe voltage the electron current collected is pro portional to exp $(\mathrm{eV} / \mathrm{kT}), V$ being the probe voltage measured relative to the plasma potential. If the logarithm of this current is plotted against voltage the slope of the resulting Iinear curve is then given by

$$
\frac{d(1 n i)}{d V}=\frac{e}{k T}=\frac{11,700}{T}
$$

A plot of $i$ versus $V$ on semi-log paper is thus seen to afiord a simple means of determining $T$, the electron temperature. Note that the total probe current measured must be corrected for the positive ion current in order to obtain the electron current. This is done in the usual manner by extrapolating the purely ionic current measured at very negative probe poten tials Into the "Boltzmann region" (cf. refexence 19, Fig.6.6). 
If the probe is made positive relative to the plasma potential then Langmuir bas shown that at sufficiently high values of probe current the square of the probe current is a linear function of the probe voltage, the slope of the $i^{2}$ versus $V$ line being

$$
S=4 A^{2} \times I \times e / \pi \mathrm{kT}
$$

where $A$ is the probe area and I the random electron cusrent density, given by

$$
I=\frac{l}{4} n e \bar{v}
$$

If Eqs. (53) and (54) are now combined and the average thermal electron velocity $\vec{v}$ replaced by $(8 \mathrm{kT} / \pi \mathrm{m})^{\frac{1}{2}}$, the electron density may be solved for:

$$
n=\frac{\pi}{A e}\left(\frac{m S}{2 E}\right)^{\frac{1}{2}}
$$

Electron density is thus determined once the slope $S$ is measured.

2. Theoretical Equations for Electron Temperature and Density.

As a cheok on the values of $T$ and $n$ as obtained from probe studies, theoretical formulas for these quantities will be used.

As regards the temperature, the following relation can be shown (44) to hold:

$$
\left(e V_{i} / \mathrm{kT}\right) \exp (\mathrm{eV} i / \mathrm{kT})=1.16(10)^{7} \mathrm{c}^{2} \mathrm{p}^{2} \mathrm{~b}^{2}
$$

Here $V_{i}$ is the ionization potential (10.38 volts), c is a constant of the gas (0.11 for mercury), and $b$ is again the radius of the discharge tube. A plot of $\mathrm{Eq}$. (56) as a function of the pressure, $p$, is shown in Fig. $\%$

An approximate formula for the electron density can be 
found by combining a number of equations derived in reference 44. For a cylindrical discharge tube (Eq. 179, Vol. II):

$$
\mathrm{n}=\mathbf{i} /\left(1.36 \otimes u b^{2}\right)
$$

The drift speed $u$ is given by (Eq. 259, VoI. I)*:

$$
u=(2 f)^{\frac{1}{4}}(\pi)^{-\frac{1}{\varepsilon}}\left(\frac{e \mathbb{E} \lambda}{m}\right)^{\frac{1}{2}}
$$

where, in turn, (Eq. I6I, Vol. II)*

$$
E=1.83 \times 10^{-4}(f)^{\frac{1}{2}} \mathrm{~T} / \lambda
$$

and $f$ represents the fraction of energy lost by an electron in a collision with a stationary gas molecule; for mercury, $f=5.5 \times 10^{-6}$. Combining all these equations, one finally obtains

$$
n=m(10)^{15} i_{d}(T)^{-\frac{1}{2}}
$$

$i_{d}$ being the discharge current in amperes.

3. Criterion for Applicability of Maxwellian Distribution

It was pointed out that the expression for the conductiv ity given in Eq. (39) holds only if the a.c. field does not exceed a certain critical value given in Eq. (36). If the field should be appreciably stronger, then the energy distribution of the electrons is no longer even approximately Maxwellian, and the resulting expressions for the conductivity (20) can only be evaluated by numerical integrations. It is thus of importance to investigate whether or not Eq. (36) was satisfied in the present experiment.

The electrical field strength inside the cavity can be 
estimated from the cavity $Q$ and from $P$, the power dissipated in the cavity. From the definition of $Q$, the energy stored in the cavity is

$$
U_{E}=Q P / W
$$

In terms of the field strength, $U_{\mathrm{E}}$ is given by

$$
U_{E}=\int_{v o l} \frac{1}{2} \epsilon E^{2} d v=\int_{0}^{a} \frac{1}{2} \epsilon\left[E_{m} J_{0}(k \rho)\right]^{2} 2 \pi \rho h d \rho=\pi a^{2} h_{1}^{2}(k a) E E_{m}^{2},
$$

where $\mathrm{E}_{\mathrm{m}}$ is the electric field along the cavity axis. Solving for $E_{m}$ and substituting the appropriate numerical values one obtains

$$
E_{m}=2.8(10)^{3}(Q P)^{\frac{1}{2}}=1.5(10)^{5}(P)^{\frac{1}{2}} \text { volts/meter }
$$

for a $Q$ of about 3000, as measured. Only a rough estimate can be made for the value of $\mathrm{P}$ : the klystron set put out about one milliwatt which was passed through approximately $20 \mathrm{db}$ of padding, giving $P=10^{-5}$ watts and hence $E_{m}=500$ volts/meter. If now the measured values of electron temperatures are substituted into Eq. (36), the critical value of field strength is found to be very noarly this same value. Since at all points off the carity axis, $E<E_{m}, E q$. (36) is seen to be at least approximately satisfied. 
Typical curves of $t_{x}$ and $\left(t_{x}^{-\frac{1}{2}}-I\right)$ taken at one mm. Hg pressure are shown in Fig. 3. Actually, the procedure followed in general was not to plot $t_{r}$, but rather to compute $\left(t_{x}^{-\frac{1}{2}}-1\right)$ for each experimental point and to plot this value directly; the resulting graphs always came out to be very nearly straight lines which are more easily fitted to somewhat scattered points. The almost linear relationship between $\left(t_{x}^{-\frac{1}{2}}-1\right)$ and discharge current is as predicted by Eqs. (12), (39), or (48) and (62), which show that

$$
\left(t_{x}^{-\frac{1}{2}}-1\right) \sim \sigma_{r} \sim n \sim i_{d}
$$

where the tilde indicates proportionality. Slight curvatures were observed, particularly in the low current region. These are probably due to a corresponding variation of electron density with current which has been observed by several investigators. Plots of frequency shift against current, such as the one shown in Fig. 4, also show the expected linear character, although again exhibiting some curvature near the origin.

By taking transmission and frequency shift data at various pressures, plots of conductivity vs. pressure with current as parameter may be obtained using Eqs. (12) and (19). Such curves are shown in Figs. 5 and 6. The real conductivity com ponent is seen to maximize at pressures around $8 \mathrm{~mm} \mathrm{Hg}$. Corc responding values of electron temperature may be calculated the data of Table XIII in reference (19). 
from this by Eq. (4Ia); they are shown in curve C, Fig. \%. Also shown there are the values of electron temperature found by probe measurements, as weII as a theoretical temperature plot using Eq. (58)。

Figuxe 6 shows a maximum occurring also in the imaginary conductivity component. Now if $\sigma_{i}$ is plotted against pressure at constant electron density, rather than at constant current, Eq. (39) or Eq. (48) predicts a monotonic decrease with presw sure starting from the maximum value of $e^{2_{n}} / m w$. Hence the observed falling off at lower pressures is apparently due to a similar decrease of electron density at these pressures. To check this assumption values of $\sigma_{r} / n$ and $\sigma_{i} / n$ were calculated from Eq. (39a), using the measured temperatures shown in Fig. 7A. By dividing these two quantities into the measured values of $\sigma_{r}$ and $\sigma_{i}$, respectively, the electron density is obtained. Curves of density calculated in this mannex are plotted in Fig. 8 for a discharge current of $100 \mu a_{\text {; }}$ the postulated decrease in density at low pressures is clearly shown. Density values obtained from probe studies are also shown in Fig. 8 and the agreement is seen to be quite good. At the large probe currents required ( $c f$. page 23 ) the discharge became unfortunately quite unstable for pressures above $2 \mathrm{~mm}$ Hg so that a complete check of the electron density curves could not be carried through. A further check on the density values of Tig. 8 is afforded by Eq. (62) which predicts electron densities varying between $5.6(10)^{9}$ and $8.0(10)^{9}$ for the electron temperatures of Fig. 7; considering the many crude assumptions 
that lead to this equation, the agreement is again quite adew quate.

As has been mentioned, the value for the electronic mean free path used in Eq. (39a) was taken from reference (43) and may well be quite inaccurate here. Fortunately, however, it is possible to determine the true m.f.p. from the conductivity measurements themselves. If the ratio $\sigma_{i} / \sigma_{r}$ is formed, then from Eq. (39) this is seen to be a function of the parameter $\mathrm{x}_{1}$ only:

$$
\sigma_{i} / \sigma_{r}=-\left(x_{1}\right)^{\frac{1}{2}} x_{3 / 2}\left(x_{1}\right) / K_{2}\left(x_{1}\right)
$$

Values of $x_{1}$ corresponding to the experimentally observed values of $\sigma_{i} / \sigma_{r}$ can therefore be found from a plot of Eq. (66), and hence the m.f.p. is determined:

$$
\lambda=\left(2 x_{1} k T / w^{2} m\right)^{\frac{1}{2}}=1.035(10)^{-5}\left(x_{1} T\right)^{\frac{1}{2}} \mathrm{~cm}
$$

Carrying through this procedure the plot of $\lambda$ versus $T$ shown in Fig. 9 is obtained. If these values of $\lambda$ are now used to re-determine the electron density then the same curve of $n$ versus pressure is, of course, obtained from both $\sigma_{r}$ and $\sigma_{i}$; it falls about midway between the curves of Fig. 8 and is shown in Fig. 10 .

Finally the electron density may also be found from the conductivity formula given in Eq. (48). The mean free time, $\tau$, must first be determined for this purpose, but this is readily done since Eq. (48) shows that

$$
\sigma_{i} / \sigma_{r}=-w \tau
$$

Using this result, Eq. (48) can be solved for $n$, giving 


$$
\mathrm{n}=1.87(10)^{12}\left(\frac{\sigma_{r}}{\sigma_{i}}+\frac{\sigma_{i}}{\sigma_{r}}\right) \sigma_{r} \text { electrons } / \mathrm{cm}^{3}
$$

The values of $n$ determined in this manner are also shown in Fig. 10 and are practically identical with those obtained above by using Eqs. (39), (66), and (67). This is of some importance, since not only is $\mathrm{Eq}$. (69) much easier to use, but it also allows a determination of electron density from the conductivity measurements alone without knowledge of the electron temperature.

The values of mean free path and mean free time between collisions are shown in Fig. 9. From these two curves the question raised in Section II-D regarding the dependence on temperature of the collision cross-section is readily answered. For if the cross-section, $q$, would decrease with increasing electron velocity then $p \lambda$ should show an upward trend in accordance with Eq. (33). Actually, however, $p \lambda$ fluctuates about a mean value of approximately $0.0095 \mathrm{~cm}$. mm $\mathrm{Hg}$; while $\mathrm{p} \tau$, on the other hand, shows a definite falling off with temperature. The conclusion to be drawn is then that $p \lambda$ and $q$ do not change over the range of electron energies encountered here. If this is true, then, since $\tau=\lambda / v, p \tau$ should be proportional to $1 / v$ or $(T)^{-\frac{1}{2}}$. Such a curve is shown on the diagram fitted to the experimental points. The fit is fairly good; an exact agreement should perhaps not be expected, since $p \tau$ was calculated from an expression which is actually not applicable in this case. 
V. FIGURES

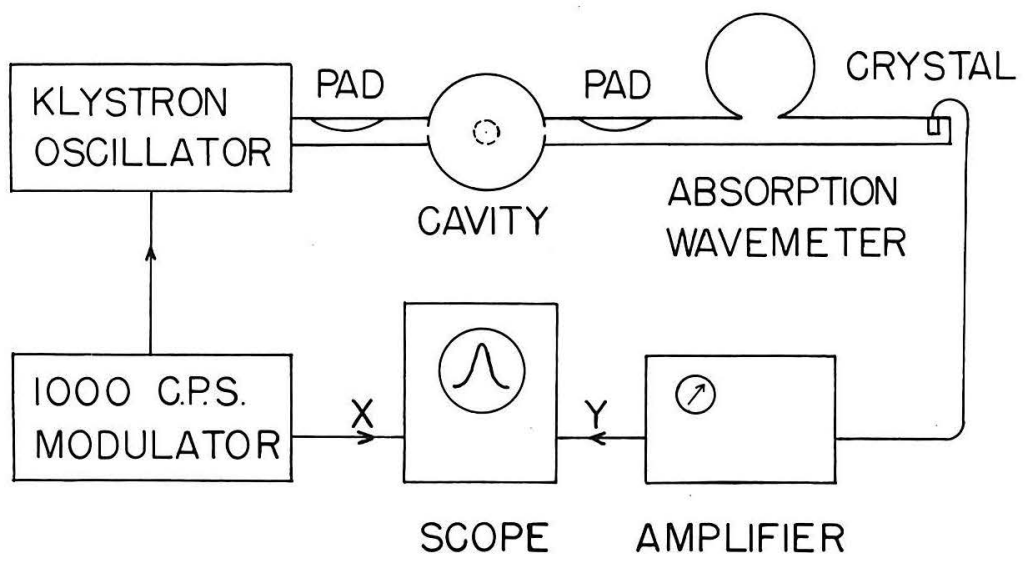

FIG. 1. Schematic diagram of experimental arrangement. 


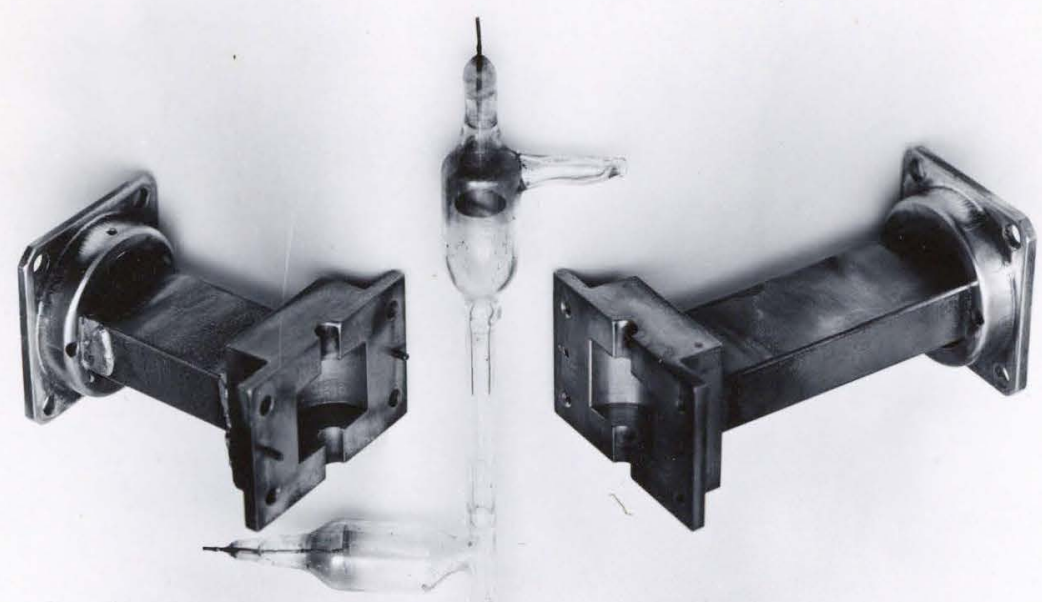

Exis

FIG. 2. Cavity and discharge tube. 


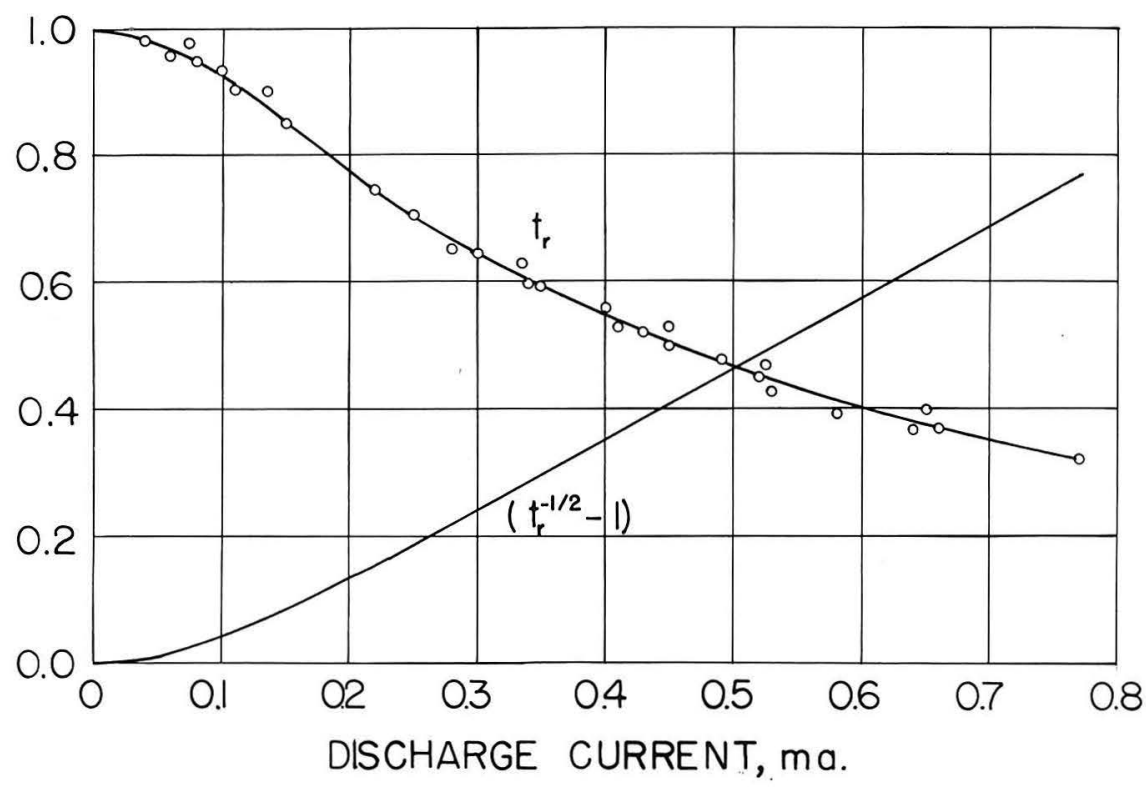

FIG. 3. Transmission characteristic at I mor Hg pressure. 


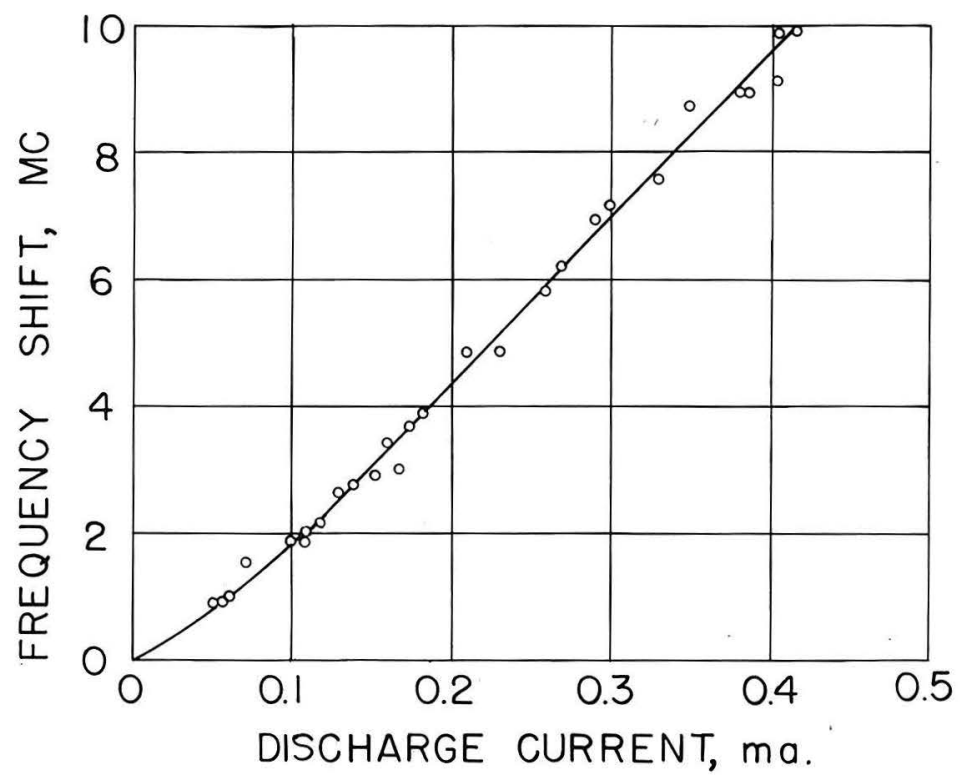

FIG. 4. Frequency shift at a mm Hg pressure. 

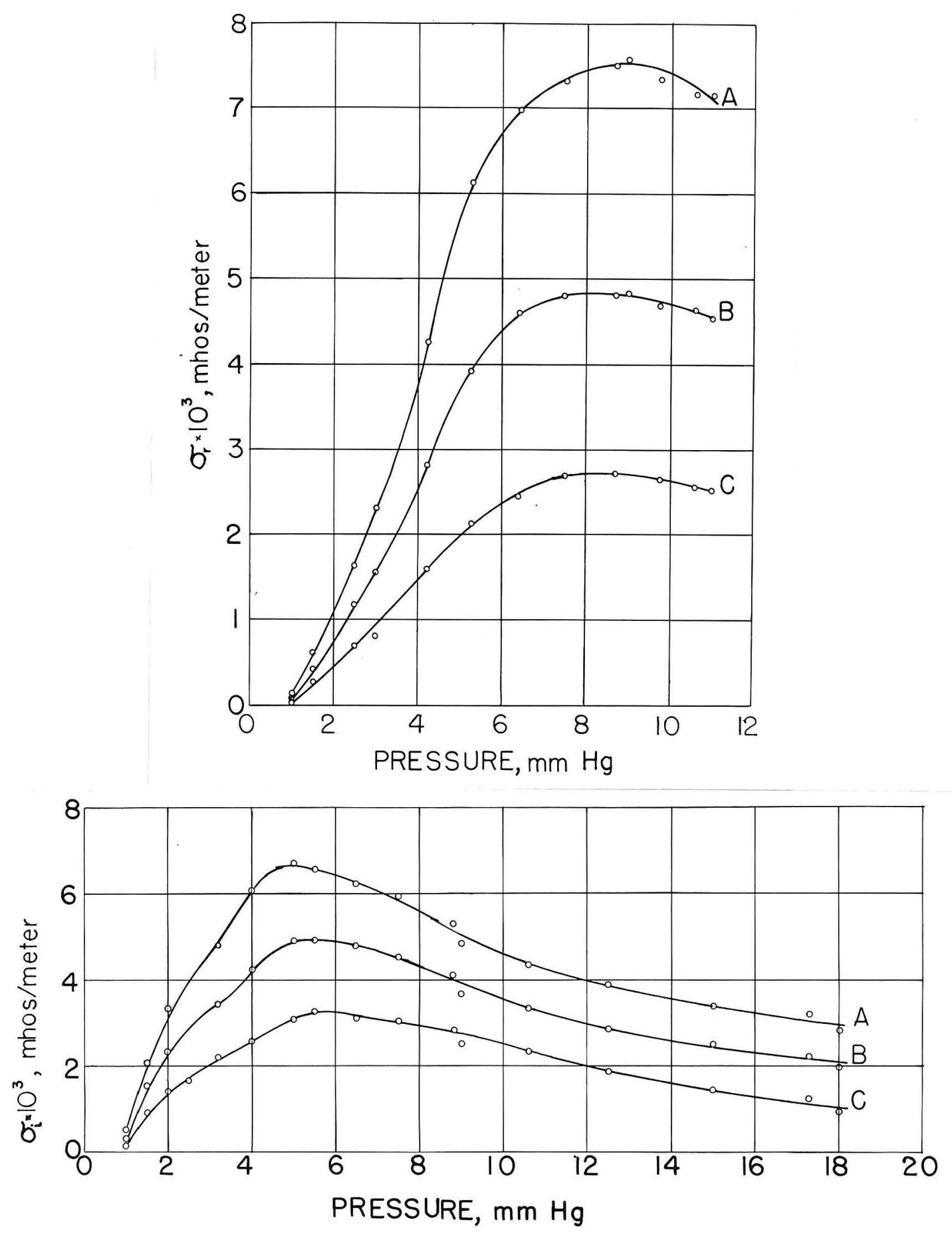

FIGS. 5 AND 6. Conductivity versus pressure: A. discharge

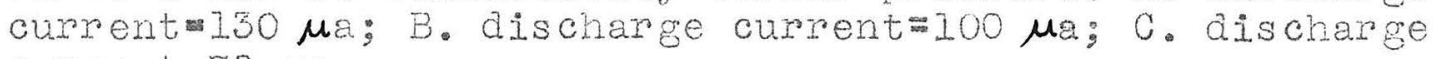
curpest $70 \mu a$. 


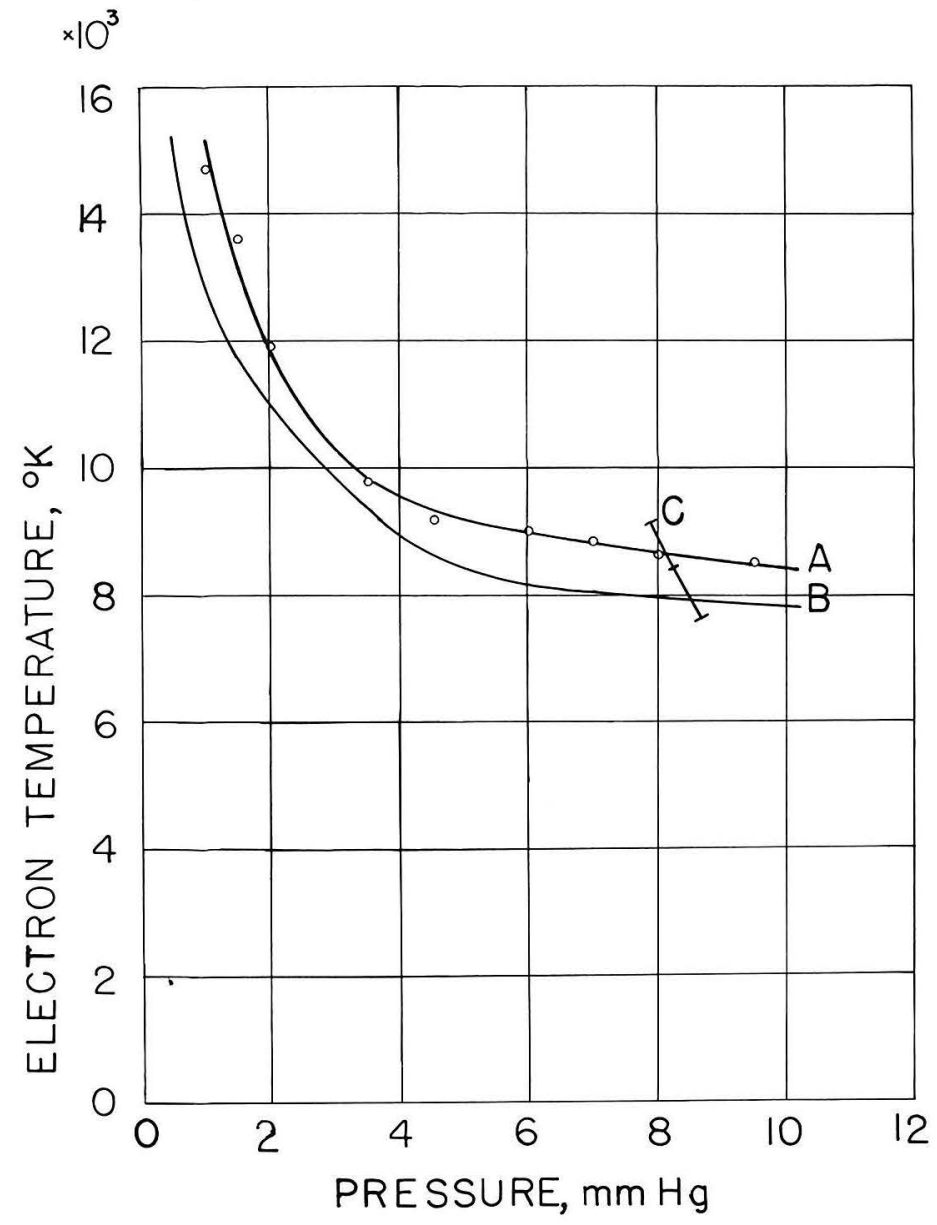

FIG. 7. Electron temperature: A. from probe studies; B. theoretical curve; C. from conductivity measurements (corresponding to $\left.7.9 \mathrm{~mm} \mathrm{Hg}<\mathrm{P}_{0}<8.7 \mathrm{~mm} \mathrm{Hg}\right)$. 


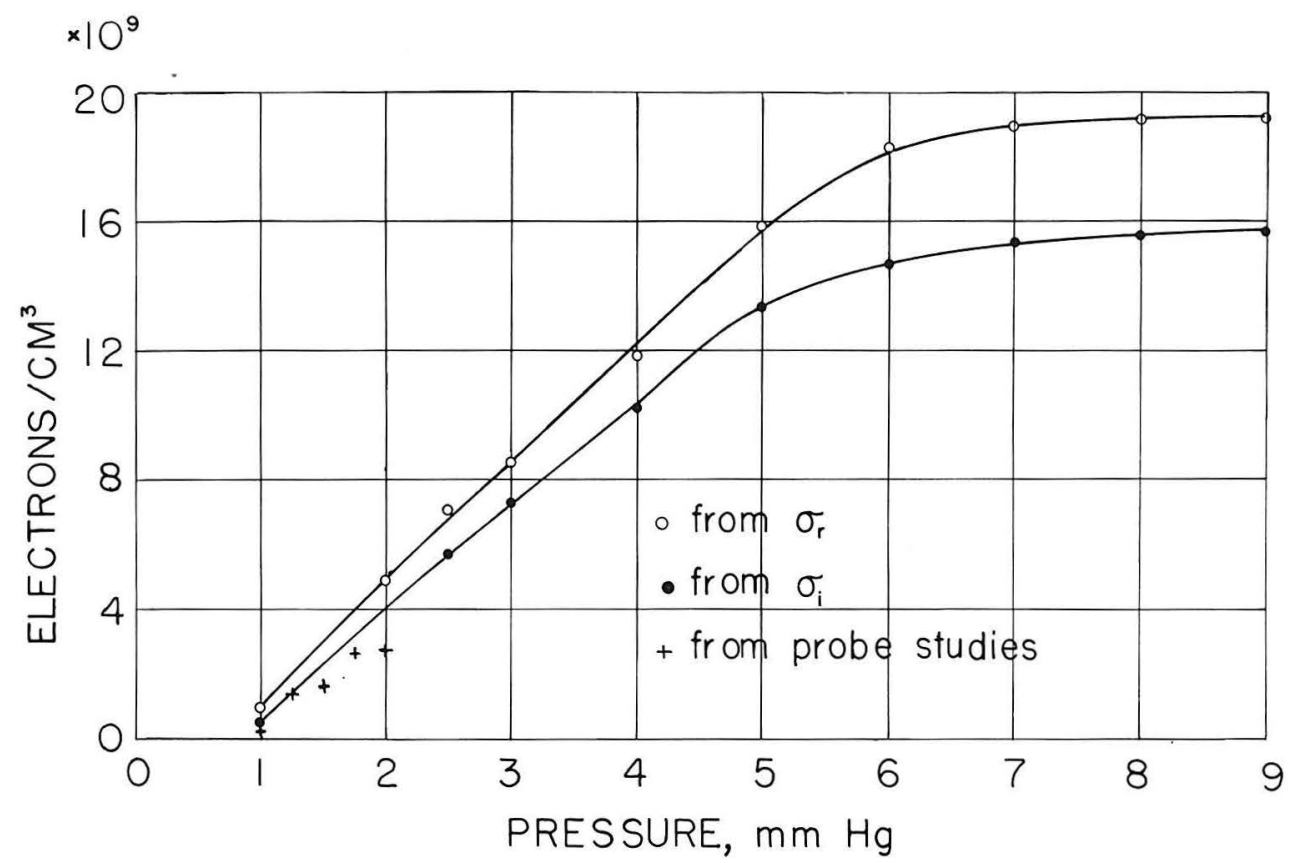

FIG. 3. Electron density versus pressure. 


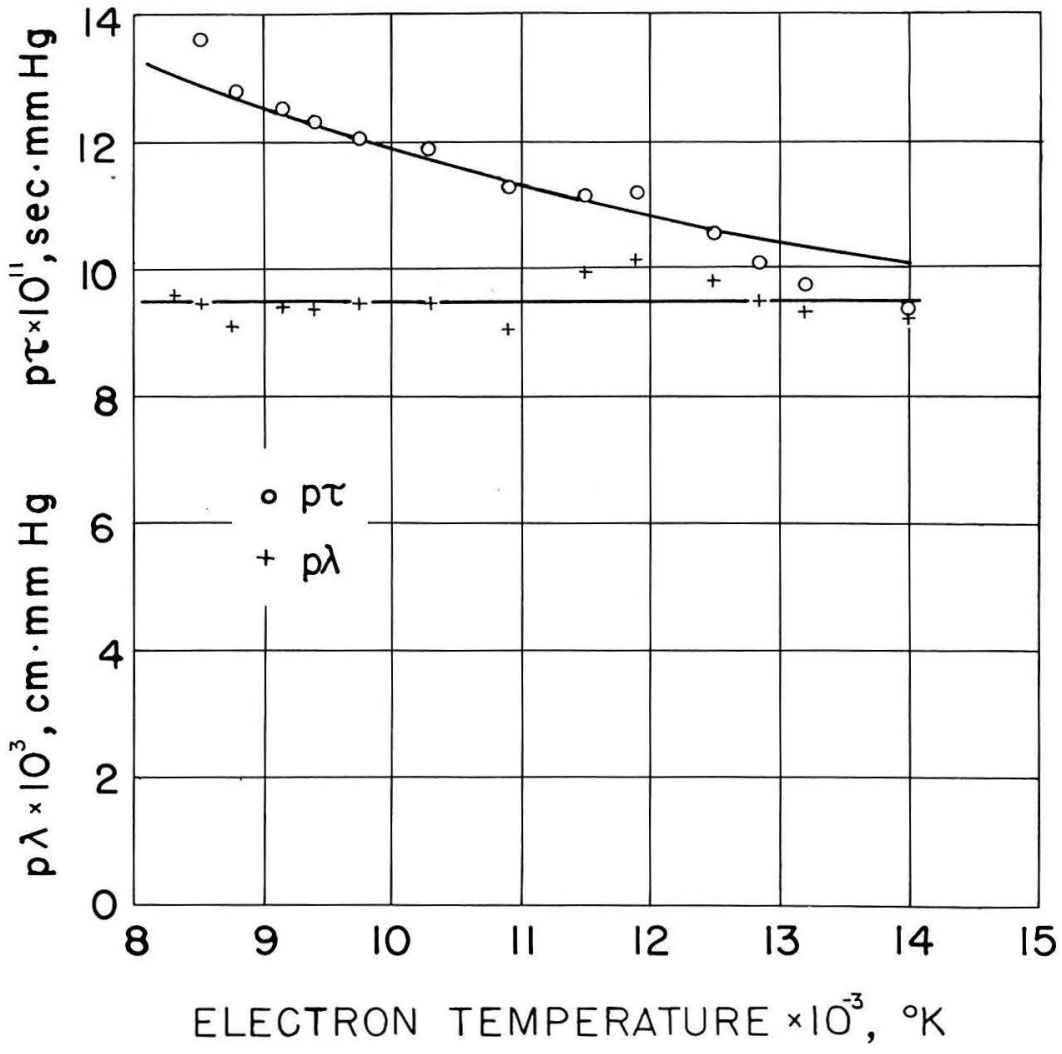

FIG. 9. Hean free path and mean free time. 


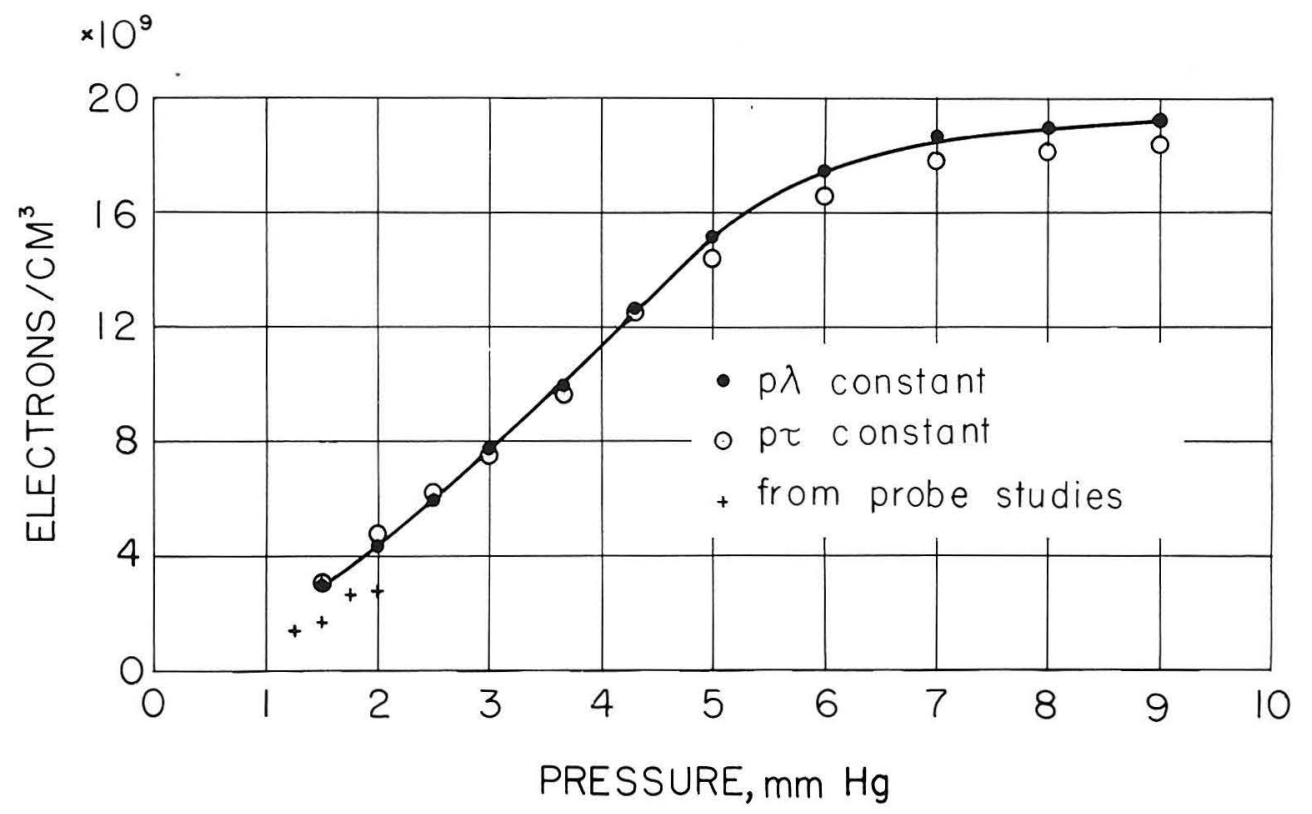

FIG. 10. Electron density. 
Pg. 39 is missing from the archival copy. 


\section{REEERENCES}

(1) Margenau, McMilian, Dearnley, Pearsall, and Hontgomery: Phys. Rev., 70, 349 (1946).

(2) I.H.R. Mimno; Rev, Mod. Phys, 9, I (1937)。

(3) F.H. Gager: "Propagation of Electromagnetic Waves Through Propellant Gases", NRL Report No. R-31.97, (1947).

(4) W.C. Hodgson, "Attenuation by Jet Exhaust", NRL Report No. R-3078, (1947).

(5) H.T. Budenbom, "Radar Effects of Missile Flame", NRI Case $26500-4$ (1948).

(6) D.E. Kerr and S.C. Brown, Buld. Am. Phys. Soc., 22, 28 (1947)。

(7) L. Goldstein and N.L. Coken, Phys, Rev, 73, 83 (1948).

(8) H.A. Prowse and B. Cooper, Nature, London, 161, 310 (1948).

(9) D.Q. Posin, Pbys. Rev., 73, 496 (1948).

(10) S.C. Brown and J.J. McCarthy, Rev. Sc. Inst, 19, 851 (1948).

(11) M.A. Herlin and S.C. Brown, Phys, Revo, 14, 291 (1948).

(12) M.A. Herlin and S.C. Brown, Phys. Rev,, 74, 910 (1848).

(13) M.A. Herlin and S.C. Brown, Phys, Rev,, 74, 1650 (1948).

(14) MacDonald and S.C. Brown, Phys. Rev,, 75, 411 (1949).

(15) M.A. Biondi and S.C. Brown, Phys. Rev., 75, 1700 (1949).

(16) A. Székély, Ann. d. Physik, 3, 112 (1929).

(17) E.V. Appleton and F.W. Chapman, Proc. Phys. Soc. (London), 44,246 (1932).

A. Székély, Acta Phys. Austriaca, 3, 22 (1949).

I. Langmuix, Gen. Elec. Rev., 27, 449, 538, 616 (1924).

H. Margenau, Phys. Rev., 69, 508 (1946).

C.K. Jen, J. Appl. Phys., 19, 649 (1948). 
(22) C.H. Collie, J.B. Hasted, and P.M. Ritson; Proc. Phys。 Soc. (London), 60, 71 (1948).

(23) G.E. Crouch, J. Chem. Phys., 16, 364 (1948).

(26) T.W. Dakin and C.N. Works, J. Appl. Phys., 18, 789 (1947).

(27) C.N.Works, J. Appl. Phys, 18, 605 (1947)。

(28) F. Borgnis, Helv. Phys. Acta, 2, 149 (1949).

(29) S.C. Brown et al., M.I.T. Research Laboratory of Electronics, Technical Report No. 66 (1948).

(30) So Ramo and J.R. Whinnery, "Fields and Waves in Modern Radioll (John Wiley and Sons, New York, 1944).

(31) J.G. Brainard et aI., "U.H.F. Techniques", (MoGrawHill Book Co., Inc., New York, 1942), p. 331 .

(32) K.K. Darrow, "Electrical Phenomena in Gases" (Williams and Wilkins Co., Baltimore, 1932), p. 200 .

(33) S. Chapman and T.G. Cowling, "The Mathematical Theory of Non-Uniform Gases" (Cambridge University Press, New York, 1939), p. 46 .

(34) H.A. Lorentz, "The Theory of Electrons" (B.G. Teubner, Leipzig, 1909), po 271.

(35) P.M. Morse, WoF.AlIis, and E.S. Lamar; Phys. Revo, 48, $412(1935)$.

(36) L.B. Loeb, "The Kinetic Theory of Gases" (McGraw-HiII Book Co., Inc, New York, 1934), p. 55.

(37) R.B. Brode, Rev。 Mod. Phys. s, 257 (1933).

(38) H. Margenau, Phys. Rev。, 73, 297 (1948).

(39) H. Margenau and L.M. Hartman, Phys. Rev., 73, 309 (1948).

(40) E.0. Hulburt, Phys. Rev。, 29, 706 (1927)。

(41) Po Langevin, Ann. Chem. Phys, 5, 245 (1905).

(42) K.T. Compton and I. Langmuix, Rev. Mod. Physo, 2, 204 (1930).

(43) J.D. Cobine, "Gaseous Conductors" (MGGraw-Hill Book Co., Inc, New York, 1941), p. 139. 
(44) von Engel and Steenbeck, "Elektrische Gasentladungen", (Verlag Julius Springer, Berlin, 1932), Vol. II, p. 85. 
VII. APPENDIX.

(Publications)

Nost of the research of this thesis has been described in an article which appeared in the Journat of Applied Physios. A brief general description of this work was also written for the periodical "Physics Today". A paper covering some gas kinetic aspects of this investigation, written jointly with Prof. Henry Margenau of Yale University, is to appear in the Physical Review later this year; only a copy of the first draft, since modified, is shown here. 
Reprinted from Journal of Applied Physics, Vol. 20, No. 11, 1125-1129, November, 1949

Copyright 1949 by the American Institute of Physics

Printed in U. S. A.

\title{
Measurement of the Complex Conductivity of an Ionized Gas at Microwave Frequencies*
}

\author{
FRED P. ADLER** \\ California Institute of Technology, Pasadena, California
}

(Received May 13, 1949)

\begin{abstract}
The positive column of a glow discharge is placed along the axis of a cylindrical cavity excited in the $\mathrm{TM}_{010}$ mode. The transmission of $3-\mathrm{cm}$ waves through the cavity and the shift in resonant frequency are observed as a function of discharge current. It is shown that from these measurements values of the complex conductivity, $\sigma_{r}+i \sigma_{i}$, of the electron gas can be calculated. Curves of the measured conductivity components as functions of pressure and current are given. Using a theoretical formula for the conductivity (see reference 6) values of electron density can in turn be calculated from both $\sigma_{r}$ and $\sigma_{i}$. Langmuir probe studies are carried out to check the results obtained, and adequate agreement is found.
\end{abstract}

\section{INTRODUCTION}

A KNOWLEDGE of the conductivity of an ionized gas is of interest in several phases of microwave work, such as the design of TR tubes, ${ }^{1}$ as well as in many problems connected with the study of the ionosphere. ${ }^{2}$ Some work has been done on discharges supported by microwave energy, ${ }^{3}$ but published measurements of conductivity $^{4}$ are restricted to frequency ranges below $10^{9}$ c.p.s.

The present paper describes a determination of conductivity at a wave-length of about $3 \mathrm{~cm}$. A d.c. mercury vapor glow discharge was used to supply the conducting gas. This has the advantage that the electron temperature and density can be determined by probe studies, using Langmuir's theory ${ }^{5}$ in conjunction with the theoretical equation for conductivity ${ }^{6}$ these measurements allow one to make a check of the values for the conductivity obtained experimentally.

* Part of a thesis to be submitted by the author to the Graduate School at the California Institute of Technology in partial fulfillment of the requirements for the degree of Doctor of Philosophy.

** General Electric Charles A. Coffin Fellow, 1947-1949.

${ }^{1}$ Margenau, McMillan, Dearnley, Pearsall, and Montgomery, Phys. Rev. 70, 349 (1946).

${ }^{2}$ L. H. R. Mimno, Rev. Mod. Phys. 9, 1 (1937).

${ }^{3}$ M. A. Herlin and S. C. Brown, Phys. Rev. 74, 291 (1948); S. C. Brown and J. J. McCarthy, Rev. Sci. Inst. 19, 851 (1948). For investigations of electrodeless discharges at lower than microwave frequencies, see: G. I. Babat, J. Inst. Elect. Eng., Part III, 27 (1947); L. Rhode, Ann. d. Physik 12, 585 (1932).

${ }^{4}$ A. Szekely, Ann. d. Physik 3, 112 (1929); E. V. Appleton and F. W. Chapman, Proc. Phys. Soc. (London) 44, 246 (1932).

${ }^{5}$ I. Langmuir, Gen. Elec. Rev. 27, 449, 538, 616 (1924).

${ }^{6}$ H. Margenau, Phys. Rev. 69, 508 (1946). For an extension of this theory, taking into account inelastic electron collisions, see also: H. Margenau, Phys. Rev. 73, 297 (1948); H. Margenau and L. M. Hartman, Phys. Rev. 73, 309 (1948); T. Holstein, Phys. Rev. 70, 367 (1946).

\section{EXPERIMENTAL APPARATUS}

A schematic diagram of the apparatus is shown in Fig. 1. The cavity used (Fig. 2) was designed to resonate near $3 \mathrm{~cm}$ in the $\mathrm{TM}_{010}$ mode. Coupling to the wave guides was through circular irises; small openings at the centers of the top and bottom walls allowed a quartz discharge tube to extend axially through the cavity. Since in the mode used the cavity wall currents fall to zero at the centers of the end plates, these apertures have no appreciable disturbing effect. The quartz tube contained a mercury glow discharge whose vapor pressure was varied between $1 \mathrm{~mm} \mathrm{Hg}$ and $18 \mathrm{~mm} \mathrm{Hg}$.

The high frequency excitation was obtained from a klystron tube frequency-modulated at a 1000-c.p.s. rate; the same modulation was also applied to the horizontal sweep of an oscilloscope. The output from the cavity was picked up by a crystal, amplified, and applied to the vertically deflecting plates of the oscilloscope. The resulting trace was thus a $Q$-curve, whose height was proportional to the transmission in power through the cavity since a square law crystal was used. A calibrated wave meter placed between cavity and crystal introduced small "pips" on the trace by means of which the

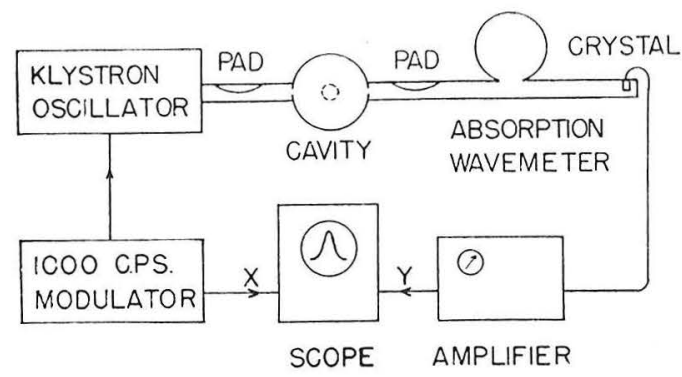

FIG. 1. Schematic diagram of experimental arrangement. 


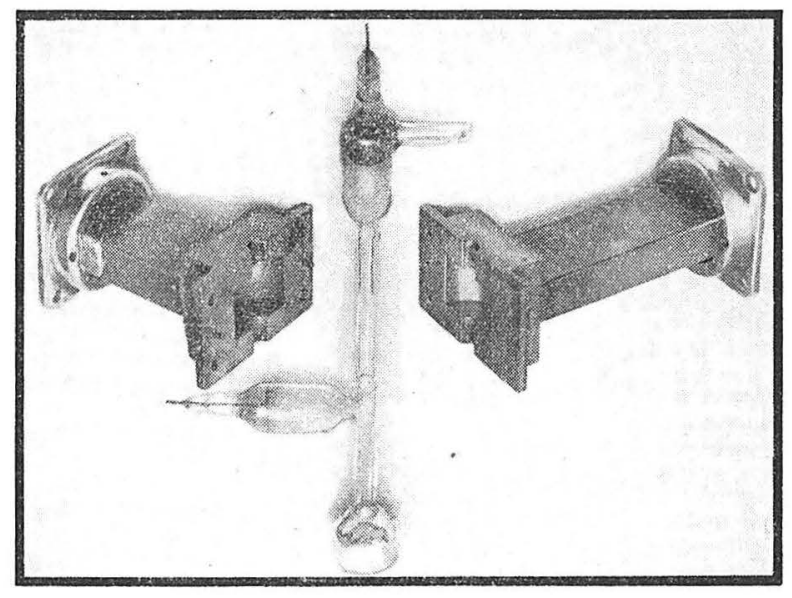

FIG. 2. Cavity and discharge tube.

resonant frequency and the $Q$ of the cavity could be measured. As shown below, measurement of these three quantities - effective $Q$, change in transmission, and change in resonant frequency with discharge currentsuffices to determine both real and imaginary components of the conductivity.

For the purpose of carrying out probe studies a second discharge tube was built identical to the one used in the cavity, except for a fine probe wire extending radially into the middle portion.

\section{THEORETICAL RELATIONS}

\section{A. Nomenclature}

t. . . . . transmission in power through the cavity, i.e., ratio of output power from the cavity to available power.

$t_{r} \ldots \ldots$ relative transmission, i.e., ratio of transmission $t$ with discharge current flowing to zero-current transmission.

$a$. . . . radius of cavity $(1.15 \mathrm{~cm})$.

$h . . .$. height of cavity $(1.5 \mathrm{~cm})$.

$b \ldots \ldots$ radius of conducting column $(0.175 \mathrm{~cm})$.

$\sigma_{r}+i \sigma_{i}$. complex conductivity of ionized mercury vapor, mhos/meter.

Q......ratio of resonant frequency to band width between half-power points; specifically the $Q$ of the unloaded cavity.

$Q_{L} \ldots \ldots Q$ of cavity with loading due to coupling to the external circuit through the irises.

$Q_{i} \ldots \ldots$ an iris loading $Q$ defined by $1 / Q_{L}=1 / Q+1 / Q_{i}$.

$Q^{\prime} \ldots \ldots Q$ of cavity loaded by the discharge (but not by irises).

$\omega_{0} \ldots . .$. nominal resonant frequency of the cavity radians/second.

$\omega_{r} \ldots \ldots$ resonant frequency with discharge current flowing.

$\Delta f \ldots$...frequency shift due to discharge in c.p.s. $=1 / 2 \pi\left(\omega_{r}-\omega_{0}\right)$.

p...... vapor pressure, $\mathrm{mm} \mathrm{Hg}$.
T.....e electron temperature, ${ }^{\circ} \mathrm{K}$.

$n$..... electron density, electrons $/ \mathrm{cm}^{3}$.

$x_{1} \ldots \ldots$ a dimensionless parameter $=\left(m \omega^{2} \lambda^{2} / 2 k T\right), \lambda$ being the electronic mean free path; for mercury at $8460 \mathrm{Mc}: x_{1}=1.2 \times 10^{6} p^{-2} T^{-1}$.

$\epsilon_{1} \ldots \ldots \epsilon_{0}\left(1+\sigma_{i} / \omega \epsilon_{0}\right) ;$ cf. Eq. (8).

$k_{1} \ldots \ldots \omega_{r}\left(\mu \epsilon_{1}\right)^{\frac{1}{2}}$.

$k_{2} \ldots \ldots \omega_{r}\left(\mu \epsilon_{0}\right)^{\frac{1}{2}}$.

k.....2.405/a.

\section{B. Calculation of the Real Component of Conductivity}

The transmission through the cavity is given $\mathrm{by}^{7}$

$$
t=\left(1-Q_{L} / Q\right)^{2}=\left(1+Q_{i} / Q\right)_{-2}^{-2} .
$$

For a cavity loaded by wall losses only, $Q$ may be expressed in terms of an equivalent shunt resistance $R$ along the cavity axis as ${ }^{8}$

$$
Q=0.00531 \stackrel{a}{h} R
$$

The small additional loading due to the presence of the quart $z$ tube can be assumed to be lumped into $R$, giving a slightly lower effective $Q$. The conducting column is now assumed to introduce an additional shunt resistance $R^{\prime}$ placed in parallel with $R$ :

$$
R^{\prime}=\frac{h}{\pi b^{2}} \cdot \frac{1}{\sigma_{r}}
$$

Thus the resultant $Q$ becomes now

$$
Q^{\prime}=0.00531 \frac{a}{h} \cdot \frac{R R^{\prime}}{R+R^{\prime}}
$$

or using (2) to substitute for $R$;

$$
Q^{\prime}=\frac{Q}{1+188 Q h / R^{\prime} a} .
$$

This then is the expression which must replace the $Q$ appearing in (1) whenever the cavity is loaded by the discharge; carrying through this substitution, and using (3) to write $R^{\prime}$ in terms of $\sigma_{r}$, the resultant expression may be manipulated into the following factored form:

$$
t=\left(1+Q_{i} / Q\right)^{-2}\left(1+592 \underset{a}{-{ }^{2}} Q_{L} \sigma_{r}\right)^{-2} .
$$

The first bracket is recognized as the transmission with no discharge current flowing; using this as a base the second bracket may be defined as a relative transmis-

${ }^{7}$ G. L. Ragan, Microwave Transmission Circuits (McGraw-Hill Book Company, Inc., New York, 1948), p. 654.

${ }^{8} \mathrm{~J}$. G. Brainerd et al., U.H.F. Techniques (McGraw-Hill Book Company, Inc., New York, 1942), p. 331. 
sion, $t_{r}$, in terms of which $\sigma_{r}$ can be expressed explicitly as:

$$
\sigma_{r}=0.00169 \frac{a}{b^{2}} \frac{1}{Q_{L}}\left(t_{r}-\frac{1}{2}-1\right) \mathrm{mhos} / \text { meter. }
$$

$l_{r}$ is readily determined, being simply the ratio of the height of the $Q$-curve trace with discharge current flowing to that with the glow turned off; for convenience and accuracy a meter was actually used which could be calibrated to read $t_{r}$ directly. Since all quantities on the right-hand side of (7) can be measured, $\sigma_{r}$ may be found.

\section{Calculation of the Imaginary Component of Conductivity}

From Maxwell's Field equation

$$
\nabla \times \mathbf{H}=\frac{\partial \mathbf{D}}{\partial t}+\left(\sigma_{r}+i \sigma_{i}\right) \mathbf{E}=\sigma_{r} \mathbf{E}+i \omega \epsilon_{0}\left(1+\frac{\sigma_{i}}{\omega \epsilon_{0}}\right) \mathbf{E}
$$

the out-of-phase conductivity component $\sigma_{i}$ is seen to result in an effective dielectric constant

$$
\epsilon_{1}=\epsilon_{0}\left(1+\frac{\sigma_{i}}{\omega \epsilon_{0}}\right)
$$

(Note: Rationalized m.k.s. units are used.) Since $\sigma_{i}$ can be shown to be negative, being due to the inertial lagging of the electrons behind the applied field, the dielectric constant is reduced, decreasing the equivalent capacity of the cavity; thus the effect of the glow discharge is to raise the resonant frequency, as shown by Eq. (12) below.

To treat the cavity as a boundary value problem, it is divided into two regions, where for $0<p<b$, $\epsilon_{1}=\epsilon_{0}\left(1+\sigma_{i} / \omega \epsilon_{0}\right)$, while for $b<\rho<a$ the dielectric constant is still $\epsilon_{0}$. No account is taken of the narrow region filled by the quartz tube which introduces a small constant frequency shift. For the inner region the expressions for the fields will be of the same form as for the usual $\mathrm{TM}_{010}$ mode:

$$
E_{z 1}=J_{0}\left(k_{1} \rho\right), \quad H_{\phi 1}=\left(\omega \epsilon_{1} / k_{1}\right) J_{1}\left(k_{1} \rho\right) .
$$

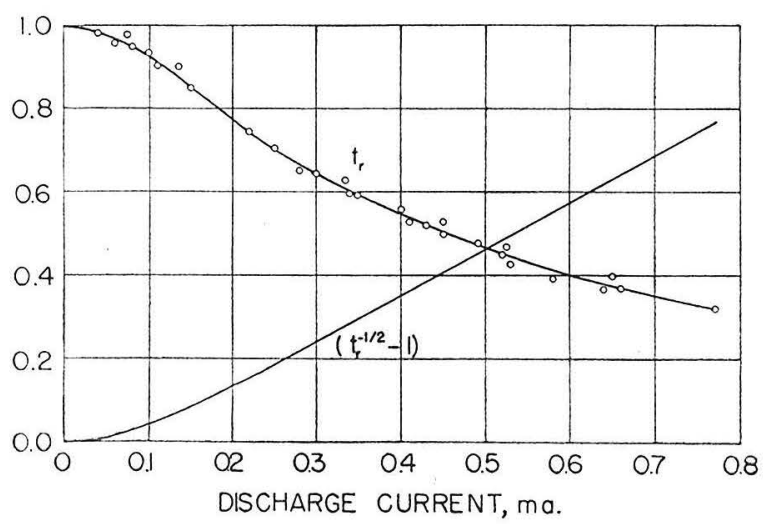

FIg. 3. Transmission characteristic at $1 \mathrm{~mm} \mathrm{Hg}$ pressure.
In the outer region Bessel functions of the second kind need also be included, so that for $b<\rho<a$ :

$$
\begin{aligned}
E_{z 2} & =A J_{0}\left(k_{2} \rho\right)+B Y_{0}\left(k_{2} \rho\right), \\
H_{\phi 2} & =\left(\omega \epsilon_{0} / k_{2}\right)\left[A J_{1}\left(k_{2} \rho\right)+B Y_{1}\left(k_{2} \rho\right)\right] .
\end{aligned}
$$

Three boundary conditions apply: at $\rho=a, E_{z 2}=0$; at $\rho=b, E_{z 2}=E_{z 1}$ and $H_{\phi 1}=H_{\phi 2}$. Using two of these to eliminate the arbitrary constants $A$ and $B$, the third one may be used to obtain the following relation which contains the desired resonant frequency implicitly in $k_{1}$ and $k_{2}$ :

$$
\frac{J_{1}\left(k_{2} b\right) Y_{0}\left(k_{2} a\right)-J_{0}\left(k_{2} a\right) Y_{1}\left(k_{2} b\right)}{J_{0}\left(k_{2} b\right) Y_{0}\left(k_{2} a\right)-J_{0}\left(k_{2} a\right) Y_{0}\left(k_{2} b\right)}=\left(\frac{\epsilon_{1}}{\epsilon_{0}}\right)^{\frac{1}{2}} \frac{J_{1}\left(k_{1} b\right)}{J_{0}\left(k_{1} b\right)} .
$$

Both sides of this equation may be linearized over a small region near $\epsilon_{1}=\epsilon_{0}, k_{1}=k_{2}, \omega_{r}=\omega_{0}$; after some numerical work this leads to the following first-order approximation for the particular case at hand:

$$
\sigma_{i}=-1.33 \times 10^{-9} \Delta f \text { mhos } / \text { meter. }
$$

An alternate method of deriving a relation between frequency shift and $\sigma_{i}$ makes use of the variation principle for electromagnetic problems (analogous to Hamilton's principle in mechanics), viz., that

$$
\iint_{\text {time }}\left(\frac{1}{2} \epsilon E^{2}-\frac{1}{2} \mu H^{2}\right) d v d t
$$

have a stationary value of zero. Since all time variations are sinusoidal, and $H$ may be replaced from Faraday's

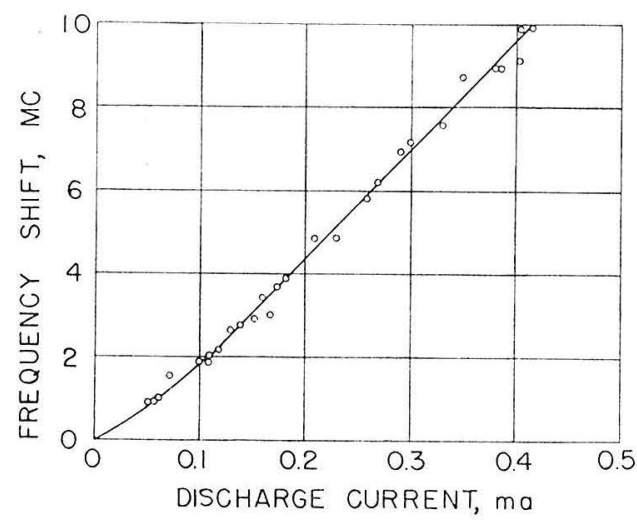

FIG. 4. Frequency shift at $2 \mathrm{~mm} \mathrm{Hg}$ pressure.

Law, $\nabla \times \mathbf{E}=-i \omega \mu \mathbf{H}$, this relation can be rewritten as

$$
\omega^{2}=\frac{\int_{01}^{1} \frac{1}{-}(\nabla \times \mathbf{E})^{2} d v}{\int_{\text {vol }} \epsilon E^{2} d v} .
$$

Assuming that the perturbation of the electric field due 

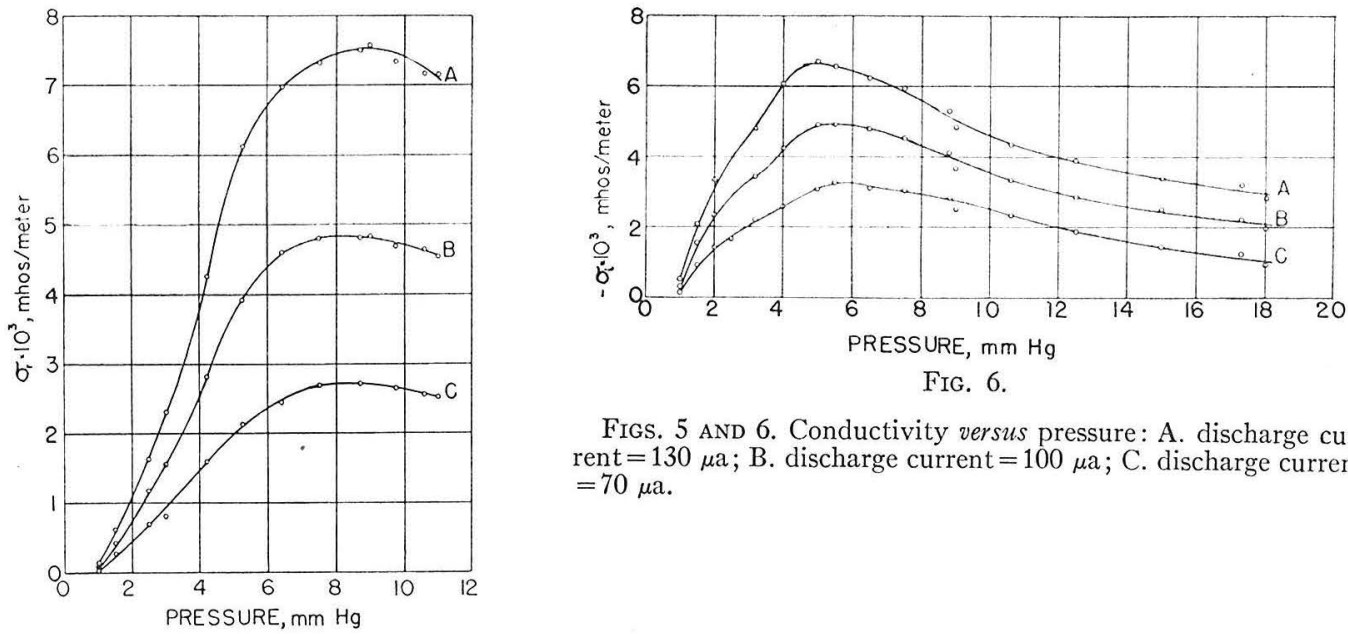

FIG. 6.

FIGS. 5 AND 6. Conductivity versus pressure: A. discharge current $=130 \mu \mathrm{a} ; \mathrm{B}$. discharge current $=100 \mu \mathrm{a} ; \mathrm{C}$. discharge current $=70 \mu \mathrm{a}$.

to the discharge is not appreciable, it is seen that the effect on the resonant frequency of any small changes in the dielectric constant can be expressed by

$$
\frac{\omega_{r}}{\omega_{0}}=\left[\frac{\int_{\mathrm{vol}} \epsilon_{0} E^{2} \cdot d v}{\int_{\mathrm{vol}} \epsilon E^{2} \cdot d v}\right]^{\frac{1}{2}},
$$

where in this case $\epsilon$ is given by Eq. (8) over the region $0<\rho<b$. Since the change in $\epsilon$ due to $\sigma_{i}$ is of the order of only 0.5 percent, one may write $1 /\left(1+\sigma_{i} / \omega \epsilon_{0}\right)^{\frac{1}{2}}$ $=1-\sigma_{i} / 2 \omega \epsilon_{0}$, obtaining:

$\frac{\omega_{r}-\omega_{0}}{\omega_{0}}=-\frac{1}{2} \frac{\int_{\mathrm{v} 01}\left(\sigma_{i} / \omega \epsilon_{0}\right) E^{2} d v}{\int_{\mathrm{v} 01} E^{2} d v}$

$$
=\frac{-\sigma_{i}}{2 \omega \epsilon_{0}} \frac{\int_{0}^{b} J_{0}^{2}(k \rho) \rho d \rho}{\int_{0}^{a} J_{0}^{2}(k \rho) \rho d \rho} .
$$

Hence

$$
\Delta f=-\frac{1}{2 \pi} \frac{\sigma_{i}}{2 \omega_{0} \epsilon_{0}}\left(\begin{array}{l}
b \\
- \\
a
\end{array}\right)^{2} \frac{J_{0}{ }^{2}(k b)+J_{1}{ }^{2}(k b)}{J_{1}{ }^{2}(k a)} .
$$

Making the proper numerical substitutions, Eq. (16) is found to agree within one percent with Eq. (12).

\section{Theoretical Expression for Conductivity}

A theoretical expression for the complex conductivity of an ionized gas has been derived by H. Margenau. ${ }^{6}$ For mercury vapor at $8460 \mathrm{Mc}$, the effective resonant fre- quency at the cavity with the tube inserted, this expression becomes: ${ }^{9}$

$$
\begin{aligned}
\sigma_{r}+i \sigma_{i}=4.38 \times 10^{-10} \frac{n}{p T^{\frac{1}{2}}} K_{2}\left(x_{1}\right) & \\
& \quad-i 4.81 \times 10^{-7} \frac{n}{p^{2} T} K_{3 / 2}\left(x_{1}\right),
\end{aligned}
$$

where

$$
K_{n}\left(x_{1}\right) \equiv \int_{0}^{\infty} \frac{x^{n} e^{-x}}{x+x_{1}} d x
$$

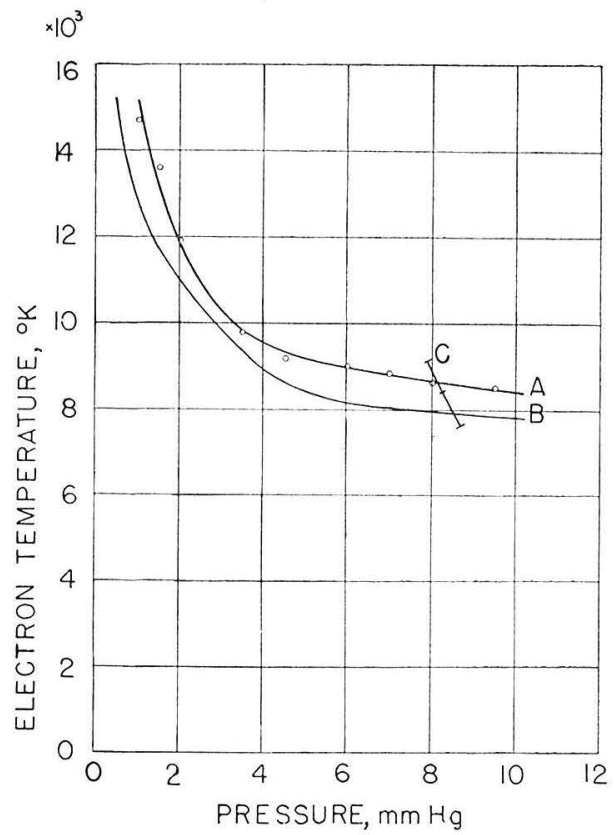

Fig. 7. Electron temperature: A. from probe studies; B. theoretical curve (see reference 11); C. from microwave measurements (corresponding to $7.9 \mathrm{~mm} \mathrm{Hg} \leq p_{0} \leq 8.7 \mathrm{~mm}$ ).

9 The value for the mean free path of mercury was taken from J. D. Cobine, Gaseous Conductors (McGraw-Hill Book Company, Inc., New York, 1941), p. 23. 
$K_{2}$ and $K_{3 / 2}$ have been plotted by Margenau. The contribution of the mercury ions can be shown to be negligible due to their large mass; hence only effects due to the electrons are actually observed.

The real component of the conductivity falls to zero at both very high and very low pressures; a maximum is predicted $^{6}$ for

$$
\omega \lambda=6.8 \times 10^{13}(k T)^{\frac{1}{2}} \text { (c.g.s.) }
$$

which corresponds very nearly to the condition of the mean free time of an electron between collisions being equal to the period of the electromagnetic wave. For mercury at the frequency used (16) becomes: ${ }^{9}$

$$
p_{0}^{2} T=570,000 \text {, }
$$

where $p_{0}$ is the pressure in $\mathrm{mm} \mathrm{Hg}$ at which $\sigma_{r}$ maximizes.

\section{EXPERIMENTAL RESULTS AND DISCUSSION}

Typical curves of $t_{r},\left(t_{r}^{-\frac{1}{2}}-1\right)$, and $\Delta f$ as a function of discharge current are shown in Figs. 3 and 4. Since $\sigma_{r}$ and $\sigma_{i}$ have been found to be proportional to $\left(t_{r}{ }^{-\frac{1}{2}}-1\right)$ and $\Delta f$, respectively, these curves show that there exists a very nearly linear relationship between conductivity and current. This same conclusion is arrived at by the analytical conductivity expression, Eq. (17), which shows $\sigma$ to be proportional to the electron density $n$, which in turn varies approximately linearly with discharge current. The slightly curved portion in the low current region is most probably due to a similar variation at $n$ with current. ${ }^{10}$

By taking transmission and frequency shift data at various pressures, plots of conductivity vs. pressure with current as parameter may be obtained through use of Eqs. (7) and (12) (Figs. 5 and 6). The real conductivity component is seen to maximize at pressures around $8 \mathrm{~mm} \mathrm{Hg}$. Corresponding values of electron temperature may be calculated from this by Eq. (19); these are shown in curve $C$, Fig. 7. Also shown there are values of electron temperature found by probe measurements as well as a theoretical temperature plot. ${ }^{11}$

Figure 6 shows a maximum occurring also in the imaginary conductivity component. Now if $\sigma_{i}$ is plotted against pressure at constant electron density, rather than constant current, Eq. (17) predicts a monotonic

\footnotetext{
${ }^{10}$ Curvatures similar to the ones observed can be seen also in plots of $n$ vs. current in the following: A. Szekely, Ann. d. Physik 3 112 (1929), Fig. 8; I. Langmuir, see reference 6, Table XIII, v. Engel and Steenbeck, Elektrische Gasentladungen (Verlag Julius Springer, Berlin, 1932), Vol, I, p. 186, Vol. II, p. 110 (1934).

${ }^{11}$ v. Engel and Steenbeck, see reference 10, Vol. II, p. 85
}

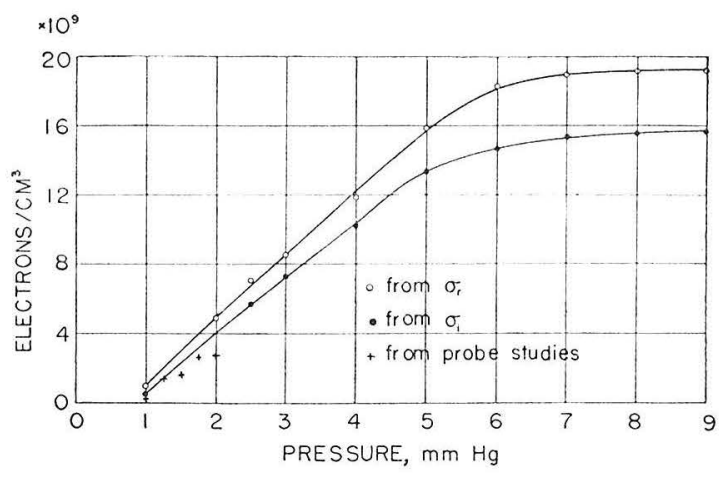

FIG. 8. Electron density versus pressure.

decrease with pressure starting from a maximum value of $e^{2} n / m \omega$. Hence the observed falling off at lower pressures is apparently due to a similar decrease of electron density at these pressures. To check this assumption values of $\sigma_{r} / n$ and $\sigma_{i} / n$ were calculated from Eq. (17), using the measured temperature shown in Fig. 7B. By dividing these two quantities into the measured values of $\sigma_{r}$ and $\sigma_{i}$, respectively, the electron density is obtained. Curves of density calculated in this manner are plotted in Fig. 8; a systematic discrepancy of about 20 percent is noticed between values found from using $\sigma_{r}$ and the ones obtained from $\sigma_{i}$. As a further check electron density was also found from probe measurements (see Fig. 8). This method involved plotting probe current squared against probe voltage; at sufficiently high values of probe current this results in a straight line from whose slope electron density may be determined. ${ }^{12}$ At the large probe currents required the discharge became unfortunately quite unstable at pressures above $2 \mathrm{~mm} \mathrm{Hg}$, so that a complete check of the electron density curves could not be carried through.

The agreement found in comparisons of experiment with theory, as shown in Figs. 7 and 8 , is probably as good as may be expected from a study of this kind.

\section{ACKNOWLEDGMENT}

The author is indebted to Dr. M. D. Fiske of the General Electric Research Laboratory who first suggested a similar study in 1946, and to Dr. J. D. Cobine, of the same laboratory, under whose guidance this project was initiated. The bulk of the work done at the California Institute of Technology under the able supervision of Professors W. H. Pickering and S. S. Mackeown.

\footnotetext{
${ }^{12}$ I. Langmuir, see reference 6, Eq. (46).
} 


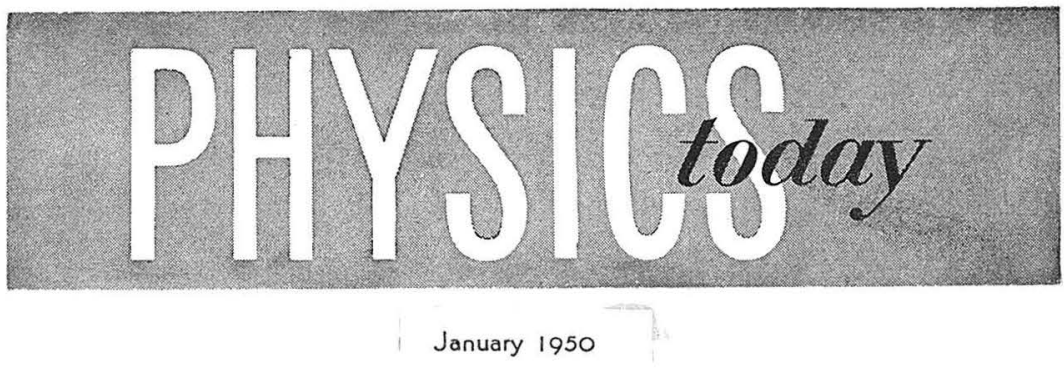

Conductivity at Microwave Frequencies

If a high frequency electric field is applied to an ionized gas, the resulting oscillatory motion of the free electrons will give rise to an alternating current. At very low gas pressures the inertia of the electrons causes this current to lag ninety degrees behind the electric field; as the pressure, and hence the density, is increased, frequent collisions of electrons with gas atoms will produce an energy loss which must be supplied by a component of current in phase with the field. This behavior may be described by assigning a complex conductivity to the ionized gas whose real and imaginary components correspond to the in-phase and out-of-phase current components. A knowledge of this conductivity is necessary in the analysis of ionosphere problems and in several phases of microwave work, such as the switching or modulation of large amounts of microwave power by means of relatively low energy gaseous discharges.

An experimental determination of complex conductivity at ten thousand megacycles has recently been made by placing a direct current glow discharge along the axis of a cylindrical microwave cavity. It was shown that both conductivity components can be calculated by determining two quantities: the relative change in transmission of three centimeter power through the cavity, and the shift in its resonant frequency, both measured as a function of discharge current and gas pressure. The experimental technique used can be extended so that high speed transient phenomena may also be investigated. Thus if the discharge current is suddenly stopped, the resultant deionization can be investigated by means of a suitable oscilloscope presentation of the output signal from the cavity, from which values of electron density versus time may be deduced. Since at the high frequencies used the relatively massive ions are not measurably affected by the microwave fields, only effects due to the free electrons are observed, leading to a great simplification in the interpretation of the results. Finally, the cavity technique may be used to study the behavior of dielectrics at microwave frequencies, simply by replacing the discharge tube by a columnar sample of the dielectric substance of interest; accurate determinations of dielectric losses can thus be made.

F.P.A.

Measurement of the Complex Conductivity of an Ionized Gas at Microwave Frequencies. By Fred P. Adler. J. $A p p$. Phys. 20: 1125, November, I949. 


\section{$-50-$ \\ Electron Conductivity and Wean Free Paths \\ by}

Fred F. Adler, California Institute of Technolosy

and

Henry Margenau, Yale University*

\section{Abstract}

lean free path and eloctron density aro calculatoci frou measurements on the complex conductivity of a discharet. Formulas besed on two altemative assumptions are toated aroinst cxperimental facts; ono is constancy of mean free path (iudependence of electron velocity), the other is constancy of mean free time.

In a rocent paperl, devoted to the moasuroment of comple

I. P. P. Haler, J. App. Fhys. 20, 1125 (1949)

electron conductivitios in a positive colum of a glov discharic, attention has been called to the opportunitios which such cxporiments hold for saining information about kinctic proccsses taking placo in the concucting gas. It was slown that olcotron densitios can be computed from the imaginary as wil as the real part of the conductivity when tho vean fros wath is given independenty, and that the donsitios arree tolsnobly woll. The purpose of the present note is to indicate how the moan freo path itself can bo detemined from such 
measuremonts, and what light this sheds on other quostions of interest in tho kinetic theory of discharges.

The formula ${ }^{2}$ used in the analysis of the exporiments

2. I. Margenau, Phys. Rov. 69, 508, (1946)

undcr discussion was based on the assurption of a constant mean froe path of tho electrons: $\lambda$ is not a function of clectron velocities $I$ and hence $\Lambda^{\text {independent of the tomore- }}$ turo, T. This is not generally true. An altemativo and oqually simple supposition is constancy of the mean erce time $\tau$, whin is $\mathrm{T} / \mathrm{v}$. What these rypotheses mean fundamontally is seen from the general relation

$$
N q \lambda=1
$$

in which $\mathbb{N}$ is the number density of moleculos intercoptirs the clectrons (in this instance $\mathrm{Hg}$ atoms) and $\mathrm{q}$ the collicion cross section, a function of $v$. It is clear that constcincy of $\lambda$ implies a constant $q$, whereas constancy of $\tau$ recuires q to be proportional to $\mathrm{v}^{-1}$. Wo shall discuss theso two altametives in sequence.

If $\lambda=$ const., the complex conductivity is given by

$$
\sigma=\frac{4}{3} \frac{e^{2} \lambda_{n}}{(2 \pi m k T)^{\frac{1}{2}}}\left[\mathrm{~K}_{2}\left(x_{1}\right)-i x_{1} \frac{1}{2} \mathrm{~K}_{3 / 2}\left(x_{1}\right)\right]=\sigma_{r}-i \sigma_{1}
$$

as was shown in reference 2 . Here $x_{1}=\frac{m w^{2}}{2 k T}$. This fomule is cxact provided the distribution function for the clectrone is haxwellian, and $T$ is the clectron temporatuo. In roference I, T was mensured by probe nethods. Tho ratio $\sigma_{i}$ is a sunction of $x_{1}$ alone, and it is plotted in that articlo. 
The experimental ratio leads therefore at once to knowledge

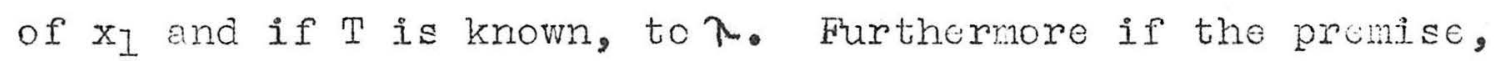
$\lambda=$ const, is true, the values thus determined should not vary with T.

The situation is somewhat different if $\tau=$ const, and the analjsis of reference 2 must be modified. Indeed the conduotivity formula is then much simpler3. Iq. 15 of the

3. Tho result we are about to derive has been clearly recognized by the workers at M.I.T., and is, in fact, impicit in some of the formulas they have used. See for instance S. S. Brown and A. D. MacDonald, Fhys. Rev. 76, 1629 (1949)

earlier paper becomes

$$
\ln f_{0}=-\frac{m / 2}{k T+\frac{M \gamma \tau^{2}}{6\left(1+\omega^{2} \tau^{2}\right)}} \int_{0}^{v^{2}} d v^{2}=\frac{m v^{2}}{2 k T^{\pi}}
$$

with

$$
T^{n}=T+\frac{1}{6 k} \cdot \frac{M r \tau^{2}}{1+\omega^{2} \tau^{2}}
$$

Eq. In stands as written, but Eq. 27 takes the form

$$
f_{1}=\frac{n \tau}{k T} \frac{1}{1+\omega^{2} \tau^{2}} f_{0}
$$

When this is inserted in Eq. 25, there results for tho curront

$$
I=\frac{n \frac{e^{2}}{m} E \tau}{1+\omega^{2} \tau^{2}}\{\cos \omega t+\omega \tau \sin \omega t\}
$$

and honce for the complex conductivity ${ }^{4}$

4. This is very similar to the Lorentz fomula for tho conductivity of electrons. It reaults if the friction constant, $G$, in the equation of motion is equated to $1 / \tau$.

$$
\sigma=\frac{n}{m} \frac{e^{2} \tau}{I+\omega^{2} \tau^{2}} \quad(1-i \omega \tau) .
$$


Here the ratio $\frac{\sigma_{i}}{\sigma_{r}}$, which is $\omega \tilde{\tau}$, leads directly to the determination of $\tau$. If the assumption $\tau=$ const. is true, the ratio test will thus reveal it. Both $\tau$ and $\lambda$ are, of course, inversely proportional to the pressure of the gas carying the discharge; as is evident from Eq. I.

In Fig. 1 the experimental data are plotted against the clectron temperature, the upper curve representing p $\tau$, the lower p $\lambda$. The pq-values were obtained by usine the relation $\sigma_{i} / \sigma_{r}=\omega \tilde{e}$, and it je seen that the uppor curvo belies its premise, the constancy of $p \tau$. However, $p \lambda$, which was computed from Eq.a, is scattered in random fashion about a constant value.

Having thus ostablished the approximate validity of our first alternative, we may use the data to dotemine the moan free path of eloctrons in $\mathrm{Hg}$. It turns out to be $9.5 \times 10^{-3} \mathrm{~cm}$ at $1 \mathrm{~mm}$ pressure.

This pro idure does not prove that $\tau$ is truly incopenden: of velocities, a result which would bo very surprisin in view of other facts. What it means is that at low electron volocities such as those to which our tomperatures corrcspond, the everage of $\lambda$ over tho range of velocities compriacd in the foxwell distribution is constant. Brode5 has measured

5. R. B. Brode, Rev. Mod. Phys. 5, 257 (1933) 
energios of 1 and 2 volts; they correspond to an average $\lambda$ between $1 / 3$ and $1 / 2$ of the value here obtained. If those inforences are to be in hamony, the collision probability mast hevo a maximum in the region where Brode's moasuroments begin and drop to low values at smaller energies.

There is some internal consistency in the two curves of Pis. 1. To be sure, the upper one jes not to be trusted. in dotcil since the theory yiolding it is not correct. But the trond is proper: if $\uparrow$ is constant, $\tau$ must decrose with $v$. Then the mean free path is determined from the retio $\sigma_{i} / \sigma_{r}$, the density of clectrons follows uniquoly fron the measurcments, and the ambiguity inherent in Firg. 8 of reference $\frac{1}{\mathscr{Z}}$ is oliminated. Fig. 2 shows the results (black dots). If tho simpler formula (3) wore chosen in analyzing tho data, the circles would result. Thus it is seen that the electron density is not very sensitive to the kinetic assumptions by which it is derived. 